Elsevier Editorial system(tm) for CARBON Manuscript Draft

Manuscript Number: CARBON-D-16-02345R1

Title: Modulated enhancement in ion transport through carbon nanotubes by lipid decoration

Article Type: Research Paper

Corresponding Author: Dr. Bing Yuan, Ph.D

Corresponding Author's Institution: Soochow University

First Author: Jiaojiao Liu

Order of Authors: Jiaojiao Liu; Bing Yuan, Ph.D; Xuewu Wu; Jingliang Li; Fangming Han; Yujiang Dou; Muzi Chen; Zhaohui Yang; Kai Yang; Yuqiang Ma

Abstract: Biomimetic channels based on carbon nanotubes (CNTs) with fast and selective transport have attractive applications in many fields. In this work, a remarkable and modulated enhancement in the ion transport rate through CNTs is facilitated by means of lipid decoration, by a factor of up to 20 times. A type of CNT membrane is firstly prepared, composed of well aligned multi-wall carbon nanotubes with an inner size of $10 \mathrm{~nm}$. An inter-diffusion method is used to efficiently incorporate lipids within the CNTs. It is found that the lipid phase state as well as the surface property of the tubes' inner walls corporately determine the assembly behavior, such as location and stability of lipids, which further influence the ion transport rate through the tubes. For example, the incorporation and self-assembly of liquid-phase DOPC and polymerized Diyne-PC within the tubes induces an enhancement in steady ion transport rate through CNTs by a factor of 5 and 20 times, respectively. In contrast, the gel-phase DPPC prefers to stay at tube tips, which increases the ion transport rate during the initial stage only. This work provides a practical guide to regulate the ion transport behaviors through CNTs for versatile applications. 
We are quite thankful for the referees' careful reviewing and thoughtful comments, which do help us think over the underlying mechanism deeply and improve the paper. The responses are detailed as follows.

\section{Response to referee 1's comments.}

Comments: The paper by Liu et al. reported a remarkable and modulated enhancement in the ion transport rate through lipid decorated CNTs. They found that the lipid phase state as well as the surface property of the tubes' inner walls corporately determine the assembly behavior, such as location and stability of lipids, which further influence the ion transport rate through the tubes. This is an interesting experimental work that could provide some guidance for separation and sensing applications. I would recommend this manuscript for publication in Carbon if the authors could satisfactorily address the following issues.

Comment 1. For the TOC, please add the unit of the rate and what the colors represent.

Response 1: (1) Color legend has been complemented in the TOC image now. (2) The histogram refers to the NORMALIZED ion transport rate through CNT-membranes before or after lipid decoration (Line 235 in the main text). Therefore, the value in y-axis represents the enhancement factor without units.

Comment 2. For the detection of lipids location, it would be better to adopt Elemental Mapping Images to reflect $\mathrm{P}$ atom distribution to highlight the decoration results.

Response 2: Thanks for the suggestion. Actually, we have tried Elemental Mapping repeatedly in our experiment. However, no signal of $\mathrm{P}$ or $\mathrm{Br}$ can be distinguished, probably due to the relatively low content of them (Fig. R1). 


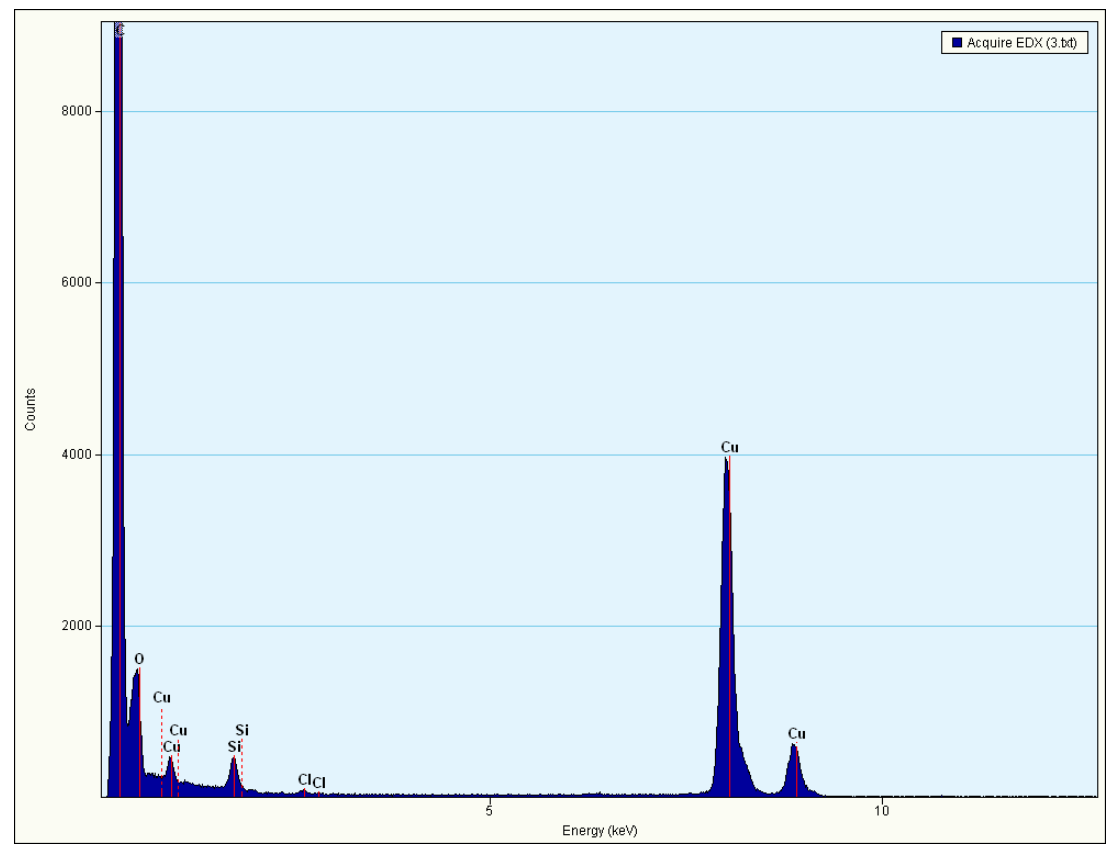

Fig. R1 Elemental mapping image of MWNTs after lipid decoration. No signal of $\mathrm{P}$ or $\mathrm{Br}$ (at 2.0 3.0 keV) was obtained. For sample preparation, the organics in the membrane was dissolved with acid etchant solution following tip sealing of the MWNTs. After that, the MWNTs were collected and supported on a copper grid for observation.

Comment 3. In the introduction, it would be better to mention that molecular simulation studies on ionic hydration confined in carbon nanotube would complement experimental studies and provide atomistic level explanations, such as, Fluid Phase Equilib, 2010, 297(2):215-220; J Phys. Chem. C, 2013, 117(21):11412-11420; ACS Nano., 2013, 7(11): 10148-10157; Nanoscale, 2014, 6(7):3686-3694; Mol. Simul., 2016, 42(10):784-798.

Response 3: Thanks for reminding. The reports mentioned by the referee were complemented as Ref. 8, 9, 14, 20 and 21, respectively, in the main text.

Comment 4. The underlying mechanism should be detailed discussed. Please add more description in explaining experimental phenomenon. What is the motivation for lipid decorated inside the MWNT to facilitate ion transport? The authors mentioned that "the strong affinity between the alkyl tails of lipid molecules and the hydrophobic aromatic plane of tubes facilitates the localization of lipids within the interior of the tubes". It would be better to discuss the connection of configuration of lipid molecules to the inner diameter of MWNT. 
Response 4: More calculations and explanations are complemented in the supporting information now. These include: (1) LJ interaction energy profiles between lipids (including lipid head and lipid tail), MWNT surface, and water, to help understand the assembly structure and stability of lipids within MWNTs (Line 294 in the main text; complemented as Section S3 in ESI). (2) Energetic analysis on DOPC and DPPC lipids in the interior of MWNTs, to explain the influence from substrate curvature on lipid assembly, and the different assembly state of the liquid-phase DOPC and gel-phase DPPC (Line 300; complemented as Section S4 in ESI).

For detailed explanations, please refer to Response 1 and 2 to the third referee.

Comment 5. The authors adopted a $\mathrm{KCl}$ solution to study the ion transport. It would be more interesting to compare the results derived from different types of ionic aqueous solution, say $\mathrm{NaCl}$ ? Does the enhancement phenomenon in the ion transport rate will be also occurred for other types of electrolyte solution?

Response 5: (1) $\mathrm{KCl}$ ions are usually used as probes to measure the ion-flux behavior of a nano-channel because the diffusion coefficients of $\mathrm{K}^{+}$and $\mathrm{Cl}^{-}$are quite close which will eliminate the liquid junction potential. Also the ion flux is easy to monitor by conductivity measurements (Hinds, B. J., Nature, 2005, 438, 44; Pan, Y. Y., J. Mater. Chem. A, 2015, 3, 11111).

(2) The transport test of $\mathrm{NaCl}$ through the DOPC-decorated MWNTs was performed and shown in Fig. R2. An enhancement is also obtained, with a factor of $\sim 2$ during both the initial and steady state.

(3) In previous reports, similarity or difference between a variety of ions was investigated. For example, diffusive transport of ions of different charge and size (e.g. $\mathrm{KCl}, \mathrm{MV}^{2+}, \mathrm{Ru}(\mathrm{bipy})_{3}{ }^{2+}$, $\mathrm{NDS}^{2-}$, and Rhod) through CNTs, which have been functionalized with carboxylic acid groups at the CNT entrance, was found to be close to bulk diffusion expectations (Hinds, B. J., ACS Nano, 2011, 5, 3867-3877). Meanwhile, ion selectivity for $\mathrm{Na}^{+}$and $\mathrm{K}^{+}$was achieved through graphene nanopores with tunable voltage (He, Z., ACS Nano, 2013, 7, 10148-10157). 

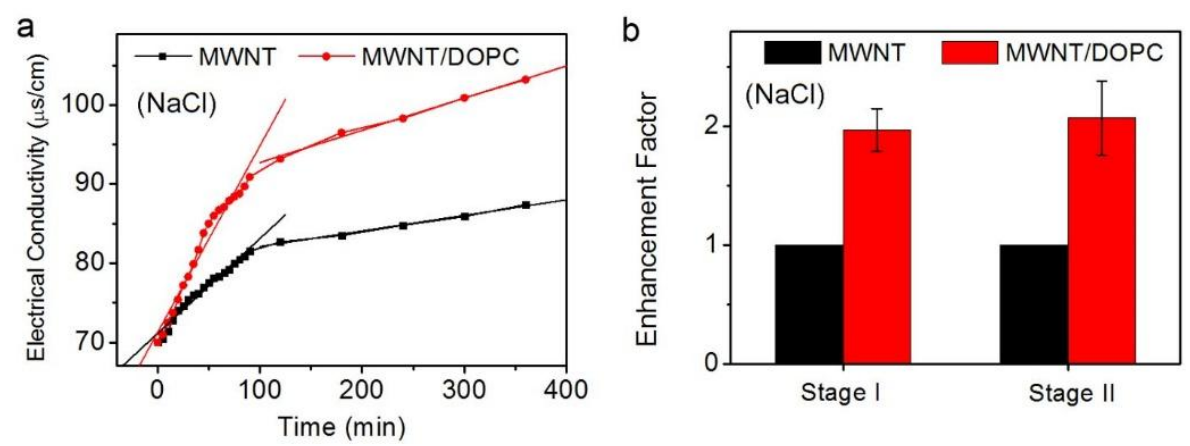

Fig. R2 Ion transport test through MWNT membranes, before and after DOPC lipid decoration, with $\mathrm{NaCl}$. (a) Electrical conductivity changes with time for the diffusion solution during $\mathrm{NaCl}$ transfer process. (b) Corresponding normalized ion transport rate. Error bars were based on the SD of 3 parallel samples. The black and red curve/histogram refer to the MWNT membrane without and with lipid decoration, respectively.

\section{Response to the second referee's comments.}

Comments: In this study, the authors have prepared two types of vertically-aligned CNT membranes composed of MWNTs or AAO-based amorphous CNTs, and investigated the influence of lipid decoration on the ion transport properties through the inner core of the nanotubes. Ion transport tests demonstrated an enhancement in the steady ion transport rate through MWNTs due to lipid incorporation, by approximately 5 times for DOPC or DPPC, and up to 20 times for Diyne-PC after polymerization. The results are very impressive and can be used to regulate the ion transport behaviors through CNTs. I have some questions and suggestions on this manuscript:

Comment 1. The authors attribute the enhanced ion transport rate of lipid decorated MWNTs to the complicate ion-lipid interactions, which could create a preferred distribution of ions near the zwitterionic lipid headgroups. I suggest the authors could decorate MWNTs by amphiphilic and electroneutral polymers, and compare the results with lipid decorated MWNTs. The additional experiments could prove if zwitterionic lipid headgroups play a key role in enhancing ion transport.

Response 1: Thanks for suggestion. Two types of molecules, i.e. PS-PAA and calcein, were used 
instead of lipid for MWNT decoration in complement experiments, to check their influence on ionic transport through tubes.

Note: PS-PAA (PS:PAA, 4.2k:5k) is a representative type of amphiphilic copolymer. It was pre-conjugated with $\mathrm{Si}$ quantum dots $(3.4 \pm 0.6 \mathrm{~nm})$ based on the carboxylic acid groups for fluorescence labeling before use. Calcein is a type of water soluble fluorescence molecule with aromatic scaffold to attach to the MWNT surface. Molecular structure of PS-PAA and calcein is shown as insets in Fig. R3.

With the same concentration-driven inter-diffusion method as lipids, PS-PAA and calcein was incorporated and localized within MWNTs, which was confirmed under confocal fluorescence observation. Ionic transport test was performed for the MWNT membranes both before and after molecular decoration. As shown in Fig. R3, the decoration of MWNT membranes with PS-PAA or calcein reduces the ion transport rate through tubes, to $\sim 60 \%$ of the initial value (during the steady state).

This result further confirms that the zwitterionic lipid headgroups play a key role in enhancing ion transport through tubes. For possible reasons, please refer to the discussion in Response 4 to the third referee.

These results were complemented as Fig. S4 in the main text and ESI. The following words were added in the main text (Line 311-313):

"Electroneutral or negatively charged molecules were also used instead of the zwitterionic lipids for MWNT decoration, which, however, reduced the ion transport rate (Fig. S4)." 

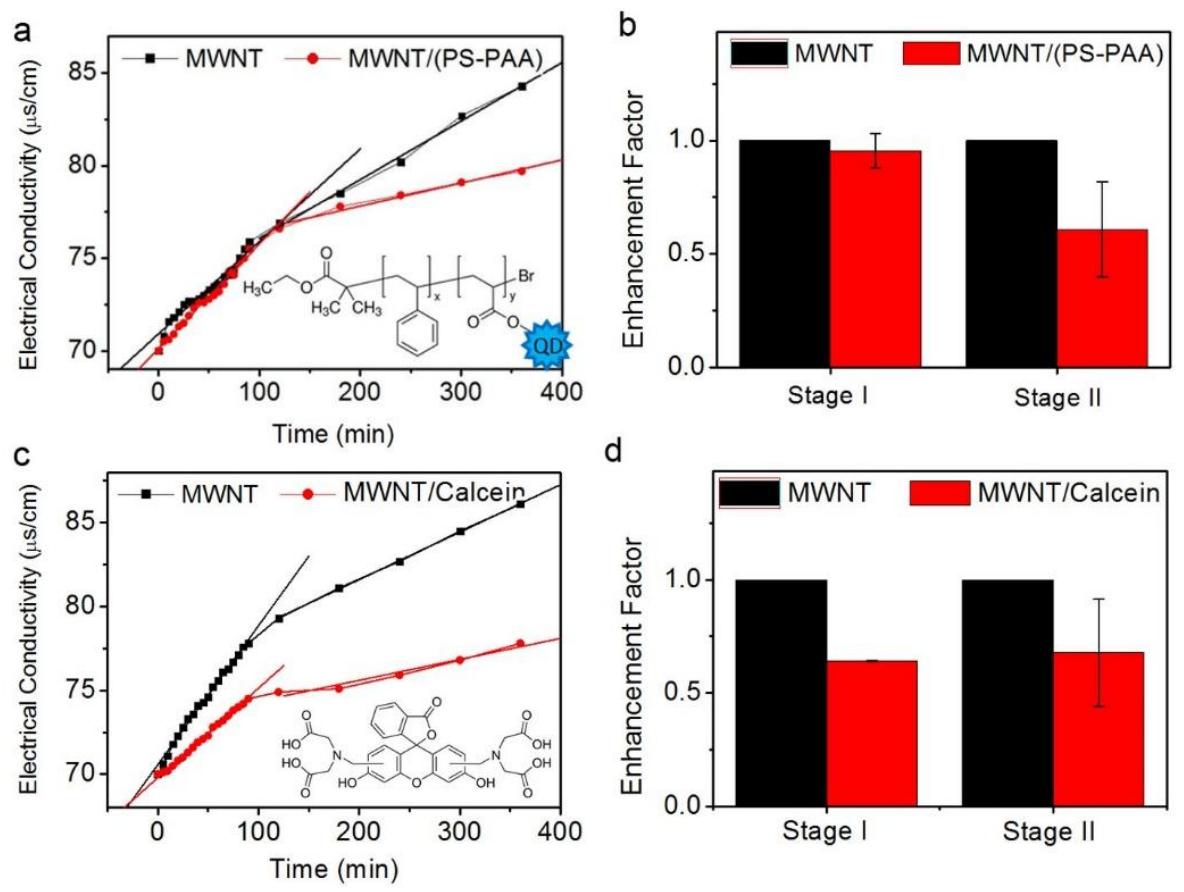

Fig. R3 Ion transport through MWNT membranes before and after molecular decoration with PS-PAA (a, b) or calcein (c, d). (a, c) Electrical conductivity changes with time for the diffusion solution during ion transfer process. (b, d) Corresponding normalized ion transport rate. Error bars were based on the SD of 3 parallel samples. The black and red curve/histogram refer to the MWNT membrane without and with molecular decoration, respectively. Molecular structure of PS-PAA and calcein was shown as insets in (a) and (c), respectively.

Comment 2. The authors compared ion transport rate of each MWNT-membrane before and after lipid decoration. How about oxidized and functionalized MWNT-membrane? Comparing different MWNT-membrane is helpful to uncover the mechanism of enhanced ion transport rate of lipid decorated MWNTs.

Response 2: The condition of oxidized or functionalized MWNT-membrane is not included in this work. There are some related publications working on it. For example: (1) It is reported that treatment of $\mathrm{CNT}$ interior with $\mathrm{HCl}$ would increase the ionic flux through tubes by a factor of over 10. (2) Diffusive transport of ions of different charge and size (e.g. $\mathrm{KCl}, \mathrm{MV}^{2+}, \mathrm{Ru}(\text { bipy })_{3}{ }^{2+}$, $\mathrm{NDS}^{2-}$, and Rhod) through CNTs, which have been functionalized with carboxylic acid groups at the CNT entrance, was investigated. The diffusion of oppositely charged ions was found to be 
close to bulk diffusion expectations of the ions, while that of similarly charged ions was reduced due to electrostatic interactions. (Hinds, B. J., Science, 2004, 303, 62-65; Hinds, B. J., ACS Nano, $2011,5,3867-3877)$

Comment 3. The inner core of MWNT-membrane is about $10 \mathrm{~nm}$, and the inner diameter of AAO-CT-membrane is about $70 \mathrm{~nm}$. The reviewer is confused on the size difference in this comparison.

Response 3: The AAO-CT-membrane was used as a control to study the influence from the "surface property of the tubes' inner walls" on assembly behavior of lipids within tubes.

Here, MWNTs have a hydrophobic inner surface and a size of $\sim 10 \mathrm{~nm}$; In contrast, AAO-CTs have a hydrophilic inner wall and a size of $\sim 70 \mathrm{~nm}$. It is noted that, compared with a highly-curved surface (e.g. tubes with a smaller size), it would be much easier for lipids to assemble and localize stably on a flatter surface (e.g. tubes with a larger size).* However, in the experiments, it is found that DOPC lipids prefer to stay in the interior of MWNTs, while they stay at tube tips of AAO-CTs. Based on the above, it can be concluded that the difference in assembly state of lipids in the MWNT- and AAO-CT-membranes is probably due to the different surface property of MWNTs and AAO-CTs.

The following words were complemented in the main text (Line 295-298):

"However, for AAO-CTs, the hydrophilic surface of the amorphous carbon layer makes it difficult for lipids to stay inside the tubes, although it is much easier for an assembled lipid layer to attach to a less curved surface (i.e. AAO-CTs with a larger size) in comparison with a highly curved one (i.e. MWNT with a smaller size; Supporting Information. Section S4).”

(*For further details, please refer to Response 1 to the third referee.)

Comment 4. Fig. 3f and Fig. 3g show the TEM images of MWNT/Diyne-PC and MWNT/Br-PC. Why are the structures not symmetric around the axis of MWNTs?

Response 4: The asymmetric distribution of lipids within CNTs is repeatedly observed under TEM. (It is speculated that such an asymmetry is as a result of the heterogeneity in the inner-wall structure of the MWNTs, or the instability of lipid assemblies on the MWNT surface.**) Although 
this, a continuous distribution of lipids along the tubes is supposed to occur, which works as a highway for the ion transport throughout the whole tube.

For accuracy, the description "homogeneous distribution of lipids within the tube" has been deleted (Line 66). Moreover, the sentence (Line 190) was changed to:

"the dark region located continuously within the cavity, indicating an uninterrupted distribution of lipids along the tube."

The sentence (Line 316-318) was changed to:

"the incorporation and continuous localization of lipids along the tubes might provide successional binding sites for ions with lipid headgroups,"

(**For more detailed explanation, please refer to Response 2 to the third referee.)

\section{Response to the third referee's comments.}

Comments: The authors prepared carbon nanotube (CNT) membranes, in which multi-wall carbon nanotubes (MWCNTs) with an inner diameter of $\sim 10 \mathrm{~nm}$ were well aligned along the membrane thickness direction. They further used a diffusion method to incorporate lipid molecules within the MWCNTs. They found that the incorporation of liquid phase DOPC and polymerized Diyne-PC lipid molecules within the tubes induced an enhancement in steady-state ion transport rate by a factor of 5 20 times. In contrast, the gel-phase DPPC preferred to stay at tube tips, and thus was only able to increase the ion transport rate during the initial stage.

I believe the experimental results are interesting. However, I have several concerns over the explanation, interpretation and discussion of their experimental results, considering, in particular, the fact that molecular modeling on the interaction between carbon nanotubes and lipid molecules has been done extensively.

Comment 1. Some simple energetic analyses should be given to explain why some lipid molecules would like to stay inside MWCNTs while others would like to stay at the ends of MWCNTs.

Response 1: For simplicity, the lipids are supposed to attach to the inner wall of MWNTs with their hydrophobic tails in the form of monolayers. According to the theoretical and simulation 
models (J. Phys. Chem. B, 2004, 108, 750-760; J. Phys. Chem. B, 2013, 117, 12113-12123), the tails of DOPC and DPPC lipids have similar affinity to the MWNT surface. However, in the interior of MWNTs, the energy cost for bending DOPC and DPPC monolayers is largely different. The explanation is as follows:

Based on Helfrich model (Huh, C., J. Soc. Pet. Eng., 1983, 23, 829; Paunov, V. N., Langmuir, $2000,16,8917-8925)$, the free energy per unit area of a cylindrical monolayer, $f$, can be taken as: $f=f_{0}-2 \kappa \frac{1}{R}+\frac{\kappa}{2} \frac{1}{R^{2}}$. Here, $\kappa$ refers to the bending modulus, $R$ is the radius of the tube, $f_{0}$ refers to the corresponding free energy of a flat monolayer, and the spontaneous curvature of the monolayer is assumed to be zero. In our case, $R$ is quite small, of approximately $10 \mathrm{~nm}$. Therefore, the value of $\Delta f=f-f_{0}$, which is mainly dominant by the term $\frac{\kappa}{2} \frac{1}{R^{2}}$, linearly increases with $\kappa$. For lipids in different phase conditions, $\kappa$ is significantly different (it is noted that the bending modulus of a monolayer approaches half of that of a corresponding bilayer, J. Chem. Theory Comput., 2013, 9, $3866-3871$ ). The $\kappa$ value of a gel-phase DPPC monolayer is roughly 10 times larger than that of a liquid-phase DOPC monolayer (Dimova, R., Advances in Colloid \& Interface Science, 2014, 208(24), 225-234; Uline, M. J., Faraday Discuss., 2013, 161, 177-303). This means that the energy cost for bending DPPC monolayer could be 10 times larger than that of a DOPC one.

Therefore, it would be much easier for the DOPC molecules to attach to the inner wall of MWNTs in comparison with the DPPC ones.

These explanations were complemented as Section S4 in the Supporting Information. The corresponding introduction was also added in the main text:

Line 298-301, "Furthermore, in comparison with gel-phase DPPC, the flexible tails of liquid-phase DOPC promotes the the assembly and localization of lipids on the highly-curved inner surface of CNTs as a result of a much lowered energy cost for layer bending (Supporting Information, Section S4)".

Comment 2. The membrane thickness is about 10 micrometers (from Fig.1c) and the inner diameter of MWCNTs is about $10 \mathrm{~nm}$. In general, DOPC lipid molecules are much smaller (a few nanometers long and sub-nanometer in diameter). In aqueous solution, lipid molecules can form 
different structures, for example, micelle or lipid bilayer. How do these lipid molecules self-assemble within the nanotubes in a homogeneous distribution? Between lipid-lipid and lipid-nanotube wall, which interaction is stronger? Answers to these questions may be important to answer how lipid molecules are distributed within the tubes.

Response 2: (1) For clarity, the statement of "homogeneous distribution" has been deleted in the main text. It is speculated that, the lipids attach to the inner wall of MWNTs with their hydrophobic tails in the form of monolayers. A continuous distribution of lipids along the tubes is supposed to occur, which works as a highway for the ion transport throughout the whole tube. However, the lipids might not homogeneously spread over the whole inner wall of MWNTs, which is confirmed by TEM images in which the structure of lipids is not symmetric around the axis of MWNTs. (Please refer to Response 4 to the second referee.)

(2) The interaction energy between lipids (including lipid head and lipid tail), CNT surface, and water, is analyzed and shown in Fig. R4 (J. Phys. Chem. B, 2004, 108, 750-760; J. Phys. Chem. B, 2013, 117, 12113-12123). Here, the minimum energy value of each profile (marked with orange arrow) is used to describe the interaction state under each condition.

It is clearly shown that, the hydrophilic lipid head prefers to stay with water ( $\mathrm{E}_{\min (\text { water-lipid }}$ head) $\left.=-5.6 \mathrm{k}_{\mathrm{B}} \mathrm{T}\right)$ while the hydrophobic lipid tail prefers to stay away from it ( $\mathrm{E}_{\min (\text { water-lipid }}$ tail $\left.=-2.0 \mathrm{k}_{\mathrm{B}} \mathrm{T}\right)$. However, for the other conditions, e.g. the interaction between lipid tail-lipid tail $\left(\mathrm{E}_{\min (\text { lipid tail-lipid tail) }}=-3.6 \mathrm{k}_{\mathrm{B}} \mathrm{T}\right)$, lipid tail-CNT $\left(\mathrm{E}_{\min (\text { lipid tail-CNT) }}=-3.2 \mathrm{k}_{\mathrm{B}} \mathrm{T}\right)$, or lipid head-CNT, water-CNT $\left(\mathrm{E}_{\min (\text { lipid head-CNT })}=\mathrm{E}_{\min (\text { water-CNT })}=-2.7 \mathrm{k}_{\mathrm{B}} \mathrm{T}\right)$, there is not much difference between them. For example, the minimum energy value between lipid head (or water) and CNT, is only $0.5 \mathrm{k}_{\mathrm{B}} \mathrm{T}$ larger than that between lipid tail and CNT, and $0.9 \mathrm{k}_{\mathrm{B}} \mathrm{T}$ larger than that between lipid tail and lipid tail. On the other hand, it is noted that the bending energy of a lipid monolayer on the highly-curved inner surface of CNTs is greatly large especially for the gel-phase DPPC monolayer (as mentioned in Response 1). Therefore, under external disturbance (e.g. drying and rehydration of the membrane during repeated tests, Line 257 in the main text), the structure of lipid monolayer within interior of CNTs might be disrupted. This might be the reason why the lipid molecules escape from the interior to outside of the tubes during repeated tests (Line 266 in the main text).

These explanations were complemented as Section $\mathbf{S 3}$ in the Supporting Information (Line 
294 in the main text).

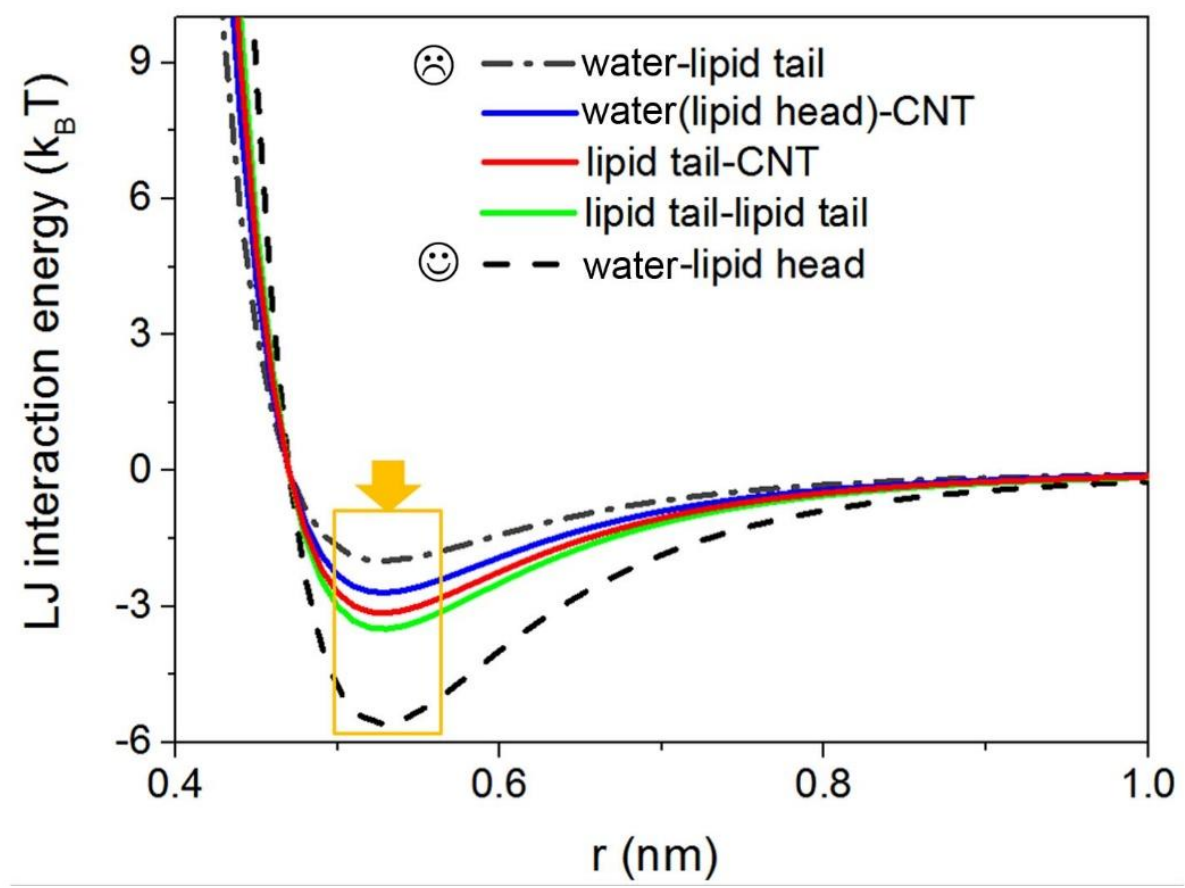

Fig. R4 LJ interaction energy distribution between different components in the system. The minimum values, marked with orange arrow, are used for comparison between different conditions.

Comment 3. The authors state that lipid molecules are stable within MWCNTs. In what form? Why? Is there any evidence to back up this statement?

Response 3: Experiments (confocal and ionic transport test) show that the localization of DOPC or DPPC lipids in interior of MWNTs is not stable during repeated tests (Line 263-266). For possible reasons, please refer to Response 2. In contrast, Diyne-PC, after polymerization, shows a good stability even after more than five cycles' test (Line 285-286).

For clarity, the statement of "stable localization" of DOPC or DPPC has been deleted in the main text (Line 294).

Comment 4. When lipid molecules are inserted into MWCNTS, polarized heads might attract ions, which in turn might exert forces to counter further ion diffusion. In addition, the presence of lipid molecules with MWCNTs might effectively reduce the cross-sectional area, which might reduce the flux of ion transport. Thus, the authors need to consider these issues to discuss what is 
going on in the initial and steady-state stages.

Response 4: Thanks for the suggestion, which makes us further think over the underlying mechanism. Ionic transport through MWNTs is determined by various factors:

(1) Steric interactions. The decrease in pore size due to molecular incorporation would probably hinder the ion transport through MWNTs. The amphiphilic and electroneutral or negatively charged (copolymer) molecules, e.g. PS-PAA and calcein which reduce the ion transport rate in our complemented experiments, are good examples to prove this (refer to Response 1 to the second referee).

(2) Electrostatic interactions. Hinds et al. reported that, functionalization of tube entrance with carboxylic groups through water-plasma oxidation, would influence the ion transport through tubes in the following manners: diffusivity of the positively charged species is close to bulk diffusion predictions, while the negatively charged species is slower than bulk diffusion predictions (Hinds, B. J., ACS Nano, 2011, 5, 3867-3877).

It is also noted that: 1' Treatment of $\mathrm{CNT}$ interior with $\mathrm{HCl}$ would increase the ionic flux through tubes by a factor of over 10 (Hinds, B. J., Science, 2004, 303, 62-65). 2' For the charge screening effect. It is influenced by the tube size as well as the ion concentration. For example, Fornaseior et al. once demonstrated salt rejection by electrostatic means in sub-2 nm CNT channels at millimolar salt concentrations (Proc. Natl. Acad. Sci., 2008, 105, 17250-17255).

(3) In our work, the diffusion situation of $\mathrm{KCl}$ through tubes with lipid decoration is quite complicated, which involves two types of ions $\left(\mathrm{K}^{+}, \mathrm{Cl}^{-}\right)$, polarized lipid headgroups, and solvent. Interaction between ions and the zwitterionic headgroups of lipids is supposed as a key factor for the enhancement of ion transport through tubes. To understand this, a simple consideration of the coulomb interaction between an electric dipole and an ion (in one-dimension case) would be helpful. As shown in Fig. R5, the interaction strength between ion and dipole (both attraction and repulsion) is significantly lower in comparison with that between ion pairs $(\oplus \leftarrow \rightarrow \oplus$,or $\ominus$ $\leftarrow \rightarrow \oplus)$. This means that it is much easier for an ion $\left(\mathrm{K}^{+}\right.$or $\left.\mathrm{Cl}^{-}\right)$to approach to or escape from a dipole, compared with a positively or negatively charged group. In this condition, the continuously distributed lipid headgroups work as a highway for the ion transport throughout the tube, which finally enhances the diffusion rate of ions. 


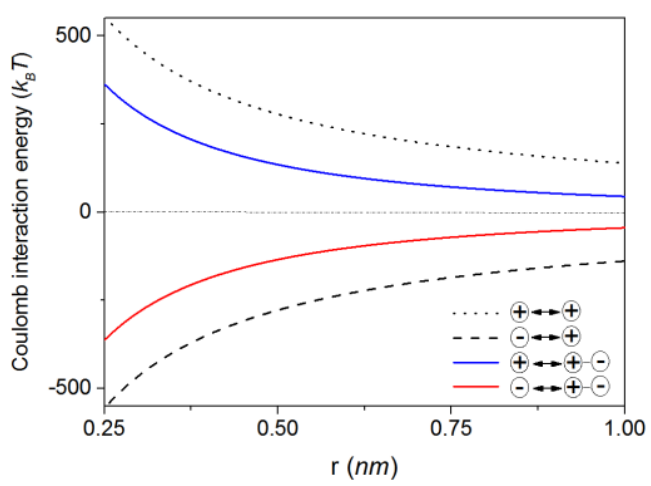

Fig. R5 Coulomb interaction energy profiles between ions and dipoles. $\oplus, \ominus$, and $\oplus-\ominus$, refer to a positively charged ion, a negatively charged ion, and a dipole, respectively.

Comment 5. For comparison, the electrical conductivity vs. time for MWCNT membrane and AAO-CT membrane without decoration of lipid molecules should be given.

Response 5: The data mentioned by the referee is provided and shown in black in Fig. 4. (Line 232-238)

Also, for the corresponding ion transport rate shown in Fig. 4-insets, the values for lipid-decorated NT-membranes are normalized by that of the naked carbon membrane. 

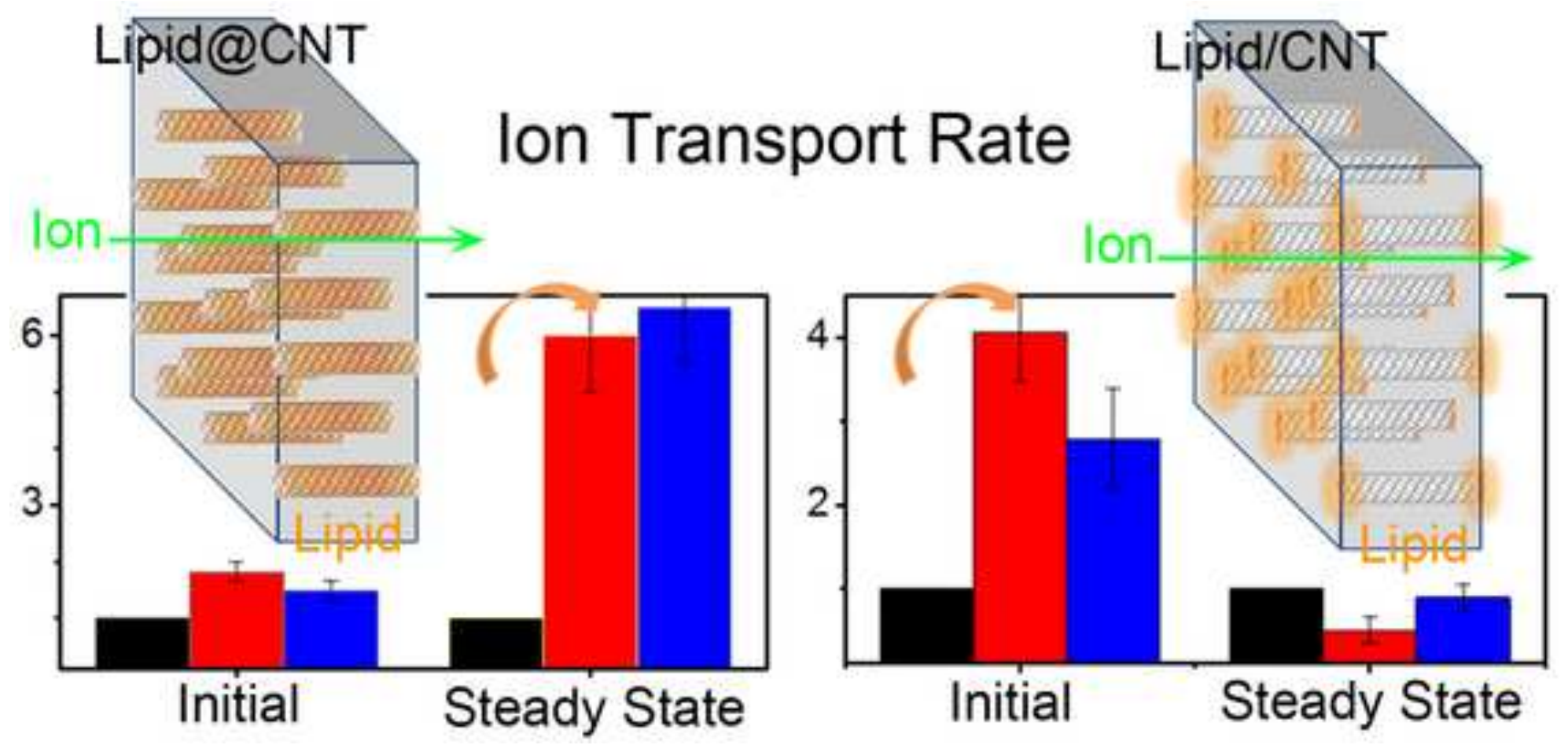

CNT

CNT+Lipid

(First test)

CNT+Lipid (Repeated test) 


\section{Modulated enhancement in ion transport through}

\section{2 carbon nanotubes by lipid decoration}

3 Jiaojiao Liu, ${ }^{\mathrm{a}, \mathrm{b}}$, Bing $\mathrm{Yuan}^{\mathrm{a}, \mathrm{b}, ~}{ }^{*}$, Xuewu $\mathrm{Wu}^{\mathrm{a}, \mathrm{b}}$, Jingliang $\mathrm{Li}^{\mathrm{e}}$, Fangming $\mathrm{Han}^{\mathrm{f}}$, Yujiang Dou ${ }^{\mathrm{c}}$, Muzi

4 Chen $^{\mathrm{d}}$, Zhaohui Yang ${ }^{\mathrm{a}, \mathrm{b},{ }^{*}}$, Kai Yang ${ }^{\mathrm{a}, \mathrm{b},{ }^{*} \text {, and Yuqiang Ma }}{ }^{\mathrm{a}, \mathrm{b}, \mathrm{g}}$

5 a Center for Soft Condensed Matter Physics and Interdisciplinary Research, ${ }^{\mathrm{b}}$ College of Physics,

6 Optoelectronics and Energy, ${ }^{\mathrm{c}}$ School of Electronic and Information Engineering, ${ }^{\mathrm{d}}$ Analysis and

7 Test Center, Soochow University, Suzhou, 215006, P. R. China.

$8 \quad{ }^{\mathrm{e}}$ Institute for Frontier Materials, Deakin University, Waurn Ponds, Vic, 3216, Australia.

$9 \quad{ }^{\mathrm{f}}$ Institute of Solid State Physics, Chinese Academy of Sciences, Hefei, 230031, P. R. China

$10 \quad{ }^{\mathrm{g}}$ National Laboratory of Solid State Microstructures and Department of Physics, Nanjing

11 University, Nanjing, 210093, P. R. China.

12 ABSTRACT. Biomimetic channels based on carbon nanotubes (CNTs) with fast and selective

13 transport have attractive applications in many fields. In this work, a remarkable and modulated

14 enhancement in the ion transport rate through CNTs is facilitated by means of lipid decoration,

${ }^{*}$ Corresponding authors.

Tel/Fax: 86512 65220239. E-mail: yuanbing@suda.edu.cn (B.Y.). Tel/Fax: 8651269156391. E-mail: yangzhaohui@suda.edu.cn $\quad$ (Z.Y.). $\quad$ Tel/Fax: $86 \quad 512 \quad 65220239 . \quad$ E-mail: yangkai@suda.edu.cn (K.Y.). 
15 by a factor of up to 20 times. A type of CNT membrane is firstly prepared, composed of well

16 aligned multi-wall carbon nanotubes with an inner size of $\sim 10 \mathrm{~nm}$. An inter-diffusion method is

17 used to efficiently incorporate lipids within the CNTs. It is found that the lipid phase state as well

18 as the surface property of the tubes' inner walls corporately determine the assembly behavior,

19 such as location and stability of lipids, which further influence the ion transport rate through the

20 tubes. For example, the incorporation and self-assembly of liquid-phase DOPC and polymerized

21 Diyne-PC within the tubes induces an enhancement in steady ion transport rate through CNTs by

22 a factor of 5 and 20 times, respectively. In contrast, the gel-phase DPPC prefers to stay at tube

23 tips, which increases the ion transport rate during the initial stage only. This work provides a

24 practical guide to regulate the ion transport behaviors through CNTs for versatile applications.

\section{1. Introduction}

26 Owing to their unique and outstanding properties, extensive research has been carried out on

27 carbon nanotubes (CNTs) for practical applications in such as novel nanomaterial science and

28 biomedical fields [1-4]. In particular, CNTs offer the potential as a candidate of mimicking

29 biological channels due to their inner-core diameter in the size range of many proteins and other

30 important biological macromolecules [5,6]. However, compared with biological protein

31 channels, which can realize extraordinarily complicated cellular functions such as selective

32 transport and high-efficient transmission of various chemicals across cell walls, the fabrication of

33 such CNT-based biomimetic channels still poses a significant challenge [7-9].

34 In fact, transport phenomena through the hollow conduits of CNTs have been attracting

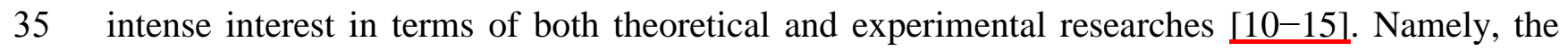

36 successful preparation of a polymer membrane composed of large quantities of CNTs arranged 
37 in parallel makes it possible for macroscopic measurement of the trans-nanotube transportation

$38[5,6,16]$. By this method, it is proven that CNTs have a distinct advantage in fluid transport with

39 four to five orders of magnitude faster than that predicted by conventional fluid-flow theory

40 [11-13]. It is assumed that such a high flow velocity is attributable to the near-frictionless

41 movement of liquid molecules along the walls of CNTs. Hydrophilic treatment can further

42 enhance mass transfer rate of CNTs. For example, by functionalizing CNTs with carboxylic acid

43 groups through plasma treatment, liquid flow through the cores of CNTs could be further

44 accelerated by $\sim 1,000-10,000$ times faster than that predicted by the conventional no-slip

45 hydrodynamic theory $[11,17]$. This finding indicates that surface modification of CNTs is a

46 powerful method to improve their mass transport capability. However, for ion transportation

47 through CNTs, the transport situation becomes much more complicated, although water is still

48 the main transport medium [18-21]. It was found that, even after plasma treatments, ion

49 diffusion through CNT was close to the bulk diffusion expectations and no obvious acceleration

50 was detected [7]. Moreover, if there are charged groups near the CNT entrance, the transport of

51 ions would be further hindered and even rejected due to the Donnan-type ion rejection

52 mechanism [16].

53 Functionalization of CNTs with phospholipids is of significance for biomedical applications

54 [22,23]. Modification of the CNT surface with PEGylated phospholipid molecules has been

55 widely used to improve the aqueous stability and biocompatibility of CNTs [24-26]. It is

56 assumed by some models that phospholipids would attach to the exterior walls of CNTs and even

57 assemble into a helical structure [27-29]. However, to the best of our knowledge, few

58 experimental studies have been reported on the assembly of phospholipids within the inner cores

59 of CNTs, and particularly its impact on the ion transportation through CNTs, which is mostly 
caused by difficulties in controllable filling of lipids into the CNT cavity and limitations in

61 characterization methods [30-34].

62 In this work, based on the millimeter-sized CNT membrane comprising well aligned multi-

63 wall carbon nanotubes (i.e., MWNT-membrane, with an inner size of $\sim 10 \mathrm{~nm}$ ), an inter-diffusion

64 method was employed to incorporate phospholipid molecules into the CNTs under mild

65 conditions. Another type of CNT membrane composed of Anodic Aluminium Oxide-based

66 carbon tubes (i.e., AAO-CT-membrane, with an inner diameter of $\sim 70 \mathrm{~nm}$ ) was used as a

67 reference. We found that lipids could stay inside the tubes or stack at their tips, depending on

68 lipid types and surface properties of the inner walls of the tubes. Ionic transport tests showed the

69 manner of lipid decoration on CNTs significantly influenced the behavior of ion transport

70 through the tubes. Incorporation of lipids in the tube interior enhances ion transport rate by a

71 factor of more than five, whereas CNT tip-exclusive lipid decoration would lead to an

72 improvement during the initial ion transport stage only. These results provide a practical guide

73 for designing advanced biomimetic nanoscale channels with controllable and high efficiency ion

74 transportation.

\section{2. Experimental Section}

2.1 Materials

78 phosphocholine (DPPC), 1-palmitoyl-2-(9,10-dibromo)stearoyl-sn-glycero-3-phosphocholine

79 (Br-PC), 1,2-di-(10Z,12Z-tricosadiynoyl)-sn-glycero-3-phosphocholine (Diyne-PC), and 1,2-

80 dipalmitoyl-sn-glycero-3-phosphoethanolamine-N-(lissaminerhodamine B sulfonyl) (Rh-PE),

81 were purchased from Avanti Polar Lipids and used as received (Fig. 1a). All other chemicals 
82 (AR) were purchased from Sinopharm Chemical Reagent Co., Ltd. and used without further 83 purification.

\section{$84 \quad 2.2$ Preparation of MWNT- and AAO-CT-membrane}

85 The MWNT-membrane was fabricated based on the preparation of a vertically-aligned MWNT-

86 array as detailed in our previous reports $[35,36]$. Briefly, a $1-10 \mathrm{~mm}$ thick MWNT-array was

87 first grown via the classical chemical vapor deposition (CVD) method on a Si wafer substrate.

88 After sealing the tubes' tips with polypropylene, ethoxyline was employed to fill the spaces

89 within the nanotube array, and this was followed by solidification. The bulk material was then

90 sliced (parallel to the substrate) using a microtome (RMC, Boeckeler Instruments, Inc.) into

91 freestanding membranes with a thickness of $10 \pm 1 \mu \mathrm{m}$. Oxygen plasma etching was then applied

92 with a PDC-32G plasma cleaner (Harrick Plasma Inc.) to remove the organic residues around the

93 tips of the CNTs ( $18 \mathrm{~W}$ for $20 \mathrm{~min}, \mathrm{O}_{2}, 500 \mathrm{mtorr}$ ). The AAO-CT-membrane was fabricated by

94 depositing a layer of amorphous carbon onto the porous AAO structure by the template method.

95 After eliminating the carbon on both surfaces of the film, an aligned carbon tube membrane with

96 penetrating pores was obtained [37].

\section{$97 \quad 2.3$ Diffusion and ion transport test}

98 Diffusion-unit set-up. The home-made diffusion unit was setup as demonstrated in Fig. 1b. The

99 unit was comprised of two cells, A and B (both in resin, each with a pore $4 \mathrm{~mm}$ in diameter), and

100 two spacers, $\mathrm{E}$ and $\mathrm{F}$ (in silicone, also had a $4 \mathrm{~mm}$ size pore in the middle). The solution in the

101 two cells could communicate through the pores and the membrane sandwiched between the 102 spacers. Each time for solution (or membrane) replacement, solutions in the two cells were 
103 poured out simultaneously, and the unit was disassembled, washed completely and dried under $104 \mathrm{~N}_{2}$ flow for its next use.

105 Ion transport test. The tests were performed based on the diffusion procedure. The membrane 106 was first installed within the diffusion unit. After that, $7 \mathrm{~mL} \mathrm{KCl}$ solution $(0.1 \mathrm{M}$ and $0.5 \mathrm{mM})$ 107 were filled into the feed and the permeate cells A and B, respectively. Thus, the total mass of $108 \mathrm{KCl}$ in the feed solution was more than two orders of magnitude greater than that in the 109 permeate, effectively eliminating depletion effects. Such a concentration gradient led to the ionic 110 transport across the membrane from A to B. The changes in electrical conductivity of the 111 permeate (cell B) were monitored simultaneously during incubation by a water analyzer 112 (Ultrameter II, Myron L Company).

113 Lipid decoration. Lipid decoration for the membrane was realized by a similar inter-diffusion 114 method. A homogeneous lipid solution $\left(0.2 \mathrm{mg} \mathrm{mL}^{-1}\right)$ was used as a mother solution. Briefly, a $115700 \mu \mathrm{L}$ lipid solution $\left(2.0 \mathrm{mg} \mathrm{mL}^{-1}\right.$, containing $0.5 \mathrm{~mol} \% \mathrm{Rh}-\mathrm{PE}$ for fluorescent labeling) was 116 transferred into an ampoule (wrapped with tin foil paper), dried under an $\mathrm{N}_{2}$ flow and kept in a 117 vacuum overnight. The membrane was then rehydrated with $7 \mathrm{~mL}$ distilled water and sonicated 118 for a complete dispersion of lipids. During lipid decoration, a naked carbon membrane was first 119 installed within the diffusion unit. $7 \mathrm{~mL}$ lipid mother solution and distilled water were added into 120 cells A and B, respectively. After incubation at room temperature for $48 \mathrm{~h}$, a fluorescent signal of 121 lipids can be detected from cell $\mathrm{B}$, which indicates that the lipids have successfully permeated 122 through the membrane. The membrane was then gently removed, washed with water and dried 123 under $\mathrm{N}_{2}$ flow for further experiments. 
124 Experiment steps. For each carbon membrane, the experiment was carried out as follows. First,

125 the naked membrane was set up for a $\mathrm{KCl}$ diffusion test in order to obtain baseline ion diffusion

126 data. Second, the membrane wad decorated with lipids, followed by other characterizations

127 including TEM and confocal imaging. Third, the carbon membrane was reassembled and step 1

128 was repeated so that the ionic diffusion rate of the membrane with incorporated lipids could be

129 measured. Fourth, the $\mathrm{KCl}$ solution was renewed, and the membrane was washed and dried, then

130 the ionic diffusion test was repeated, as described in the main text. Specifically, for lipid Diyne-

131 PC, after step 3, the carbon membrane was removed, dried and placed on a glass slide under UV

132 exposure for $30 \mathrm{~min}$ ( $285 \mathrm{~nm}$ wavelength) for lipid polymerization. Finally, the membrane was

133 reassembled for the following ionic diffusion test.

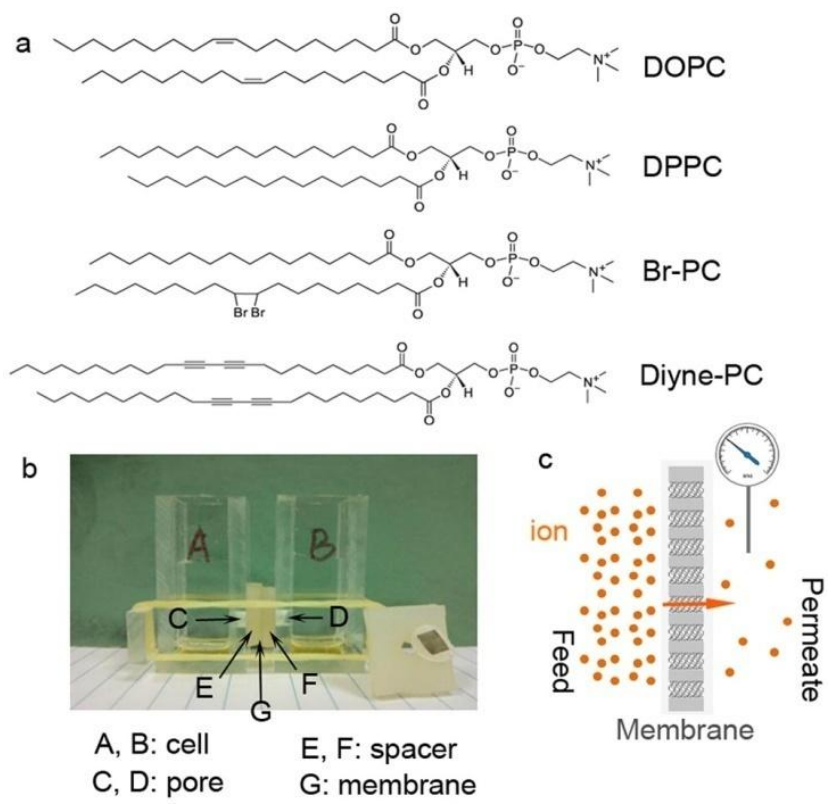

135 Fig. 1 Chemical structures of lipids and images demonstrating the diffusion unit and process. (a)

136 Molecular structure of lipids DOPC, DPPC, Br-PC and Diyne-PC. (b) Digital photo of the

137 diffusion unit, including cells (A, B) with pores on one side (C, D), spacers (E, F) with pores,

138 and the sandwiched membrane (G; cannot be seen here). Another spacer (and membrane) was 
139 placed aside for reference. (c) Schematic showing the transport of ions through CNTs within a

140 membrane from the feed side to the permeate side.

\section{$141 \quad 2.4$ Characterizations}

142 The morphology of the carbon membranes was characterized with SEM (Hitachi S-4700,

143 Hitachi). The structure of the lipids within the membrane was further characterized with TEM

144 (FEI Tecnai G-20) at $200 \mathrm{kV}$ and small angle X-ray scattering (SAXS) at Shanghai Synchrotron

145 Radiation Facility (SSRF).

146 Optical observation was performed on an inverted confocal laser scanning microscope (LSM

147 710, Zeiss) equipped with a 100× oil objective. Rhodamine-conjugated phospholipids were

148 excited by a He-Ne laser (EX $543 \mathrm{~nm}$ ), and the fluorescence was observed through filter set 20

149 (EM BP 575-640 nm). In the meantime, the transmission channel illuminated with a halogen

150 lamp was acquired. All experiments were carried out at room temperature.

\section{3. Result and discussion}

\section{$152 \quad 3.1$ Characterization}

153 Fig. 2 shows schematic and SEM images of the as-fabricated MWNT- and AAO-CT-

154 membranes. For a MWNT-membrane with a thickness of $10 \pm 1 \mu \mathrm{m}$, the nanotubes, which are

155 normal-oriented and parallel-aligned in the membrane, are clearly distinguishable from the cross-

156 sectional image under SEM (Fig. 2b, c). On the other hand, for the AAO-CT-membrane, the

157 carbon tubes with similar orientation and alignment have a much larger pore diameter of $\sim 70 \mathrm{~nm}$

158 and a length of $43 \pm 4 \mu \mathrm{m}$. It is worth noting that the nanopores of CNTs are the only paths for

159 mass transportation due to the impermeable polymer matrix (or AAO template). Thus, the 
161 the transport through the inner cores of both types of CNTs [35].

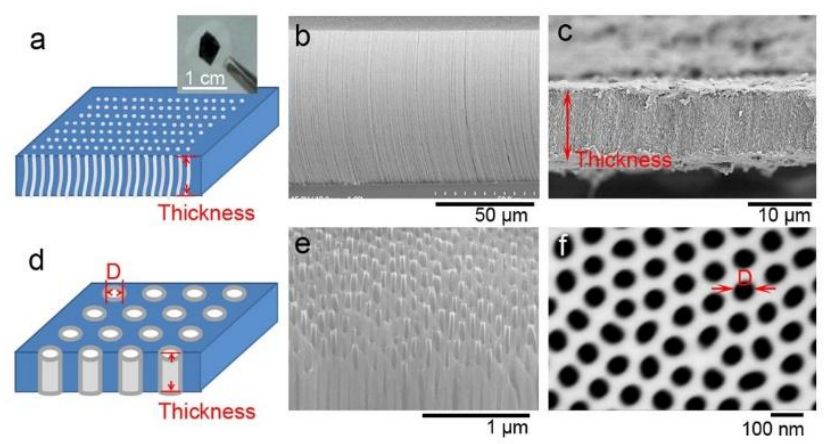

163 Fig. 2 Schematic and SEM images of the MWNT- (a-c) and AAO-CT- (d-f) membranes. (a)

164 Schematic, (a-inset) digital and (c) cross-sectional SEM images of a MWNT-membrane. (b)

165 presents the aligned MWNT array grown vertically on a substrate. (d) Schematic, (e) cross166 sectional and (f) top-view SEM images of an AAO-based amorphous CT-membrane.

168 The membrane was then sandwiched within the diffusion unit for lipid decoration and the 169 subsequent ion diffusion test. Fig. 3 shows confocal fluorescence images of the MWNT- and 170 AAO-CT-membranes right after DOPC decoration. The membrane surfaces and location of the 171 fluorescent lipids can be distinguished from the transmission and red fluorescence channels, 172 respectively. Moreover, based on the three dimensional (3D) scanning, it was observed that for 173 the MWNT-membrane, the lipids were located within the interior of the CNTs (Fig. 3b, i). 174 However, for the AAO-CT-membrane, the lipids were uniformly distributed on the two ends of 175 the CNTs, with an inter-layer distance similar to the thickness of the initial AAO-CT-membrane 176 (Fig. 3d). Z-stack images of both membranes are shown for reference in Fig. S1 in the supporting 177 information. Here, DOPC is replaced with various other types of lipids, including DPPC (in gel 
178 phase at room temperature), Diyne-PC (a kind of diacetylene phospholipid) and Br-PC (labeled

179 with two $\mathrm{Br}$ atoms in each molecule). All the above-mentioned lipids share similar assembly 180 states of CNTs with DOPC.

181 To obtain more details of the assembly method of lipids within CNTs, the scaffold (polymeric 182 or AAO) membranes were digested and the CNTs (with loaded lipids) were redispersed and 183 loaded on a lacey support membrane for TEM imaging. To have a good contrast under TEM, Br184 PC and Diyne-PC were used here instead of DOPC or DPPC. Fig. 3e-g show TEM images of the 185 MWNTs (with an inner core diameter of $\sim 10 \mathrm{~nm}$ ) without and with lipid decoration, from which 186 the multilayer structure of the tube walls can be obviously distinguished (Arrow 1). It should be 187 noted that for the native MWNTs, the cavity region is much dimmer in comparison with the wall 188 region. However, for the lipid-decorated tubes, the cavity region is even darker in contrast with 189 the walls (Arrow 3). This is reasonable considering that, compared with $\mathrm{C}$, the $\mathrm{P}$ (and/or $\mathrm{Br}$ ) 190 atoms from the lipids contribute a much stronger influence on the electron beam during TEM 191 imaging. This result further confirms the existence of lipids within the MWNTs (although a gap 192 might appear somewhere between the wall and the encapsulated lipid molecules as indicated 193 with Arrow 2) [37]. Furthermore, the dark region located continuously within the cavity, 194 indicating an uninterrupted distribution of lipids along the tube. In contrast, similar morphologies 195 were obtained for the AAO-CT membranes without and with lipid decoration. The inner core 196 region is much dimmer than the wall region, indicating that hardly any lipid remains inside the 197 amorphous carbon tubes (Fig. 2h). Synchrotron X-ray scattering was also carried out to 198 characterize the lipid structure within both membranes. However, only signal of an ordered 199 structure with a period of $\sim 155 \mathrm{~nm}$ (Fig. S2), probably referring to the parallel-distributed 200 amorphous carbon tubes (i.e. porous aluminum template), was acquired from the AAO-CT- 
201 membrane. No signal of ordered lipid structures (such as lamellar or hexagonal assembly) was

202 acquired from either membrane (Supporting Information).
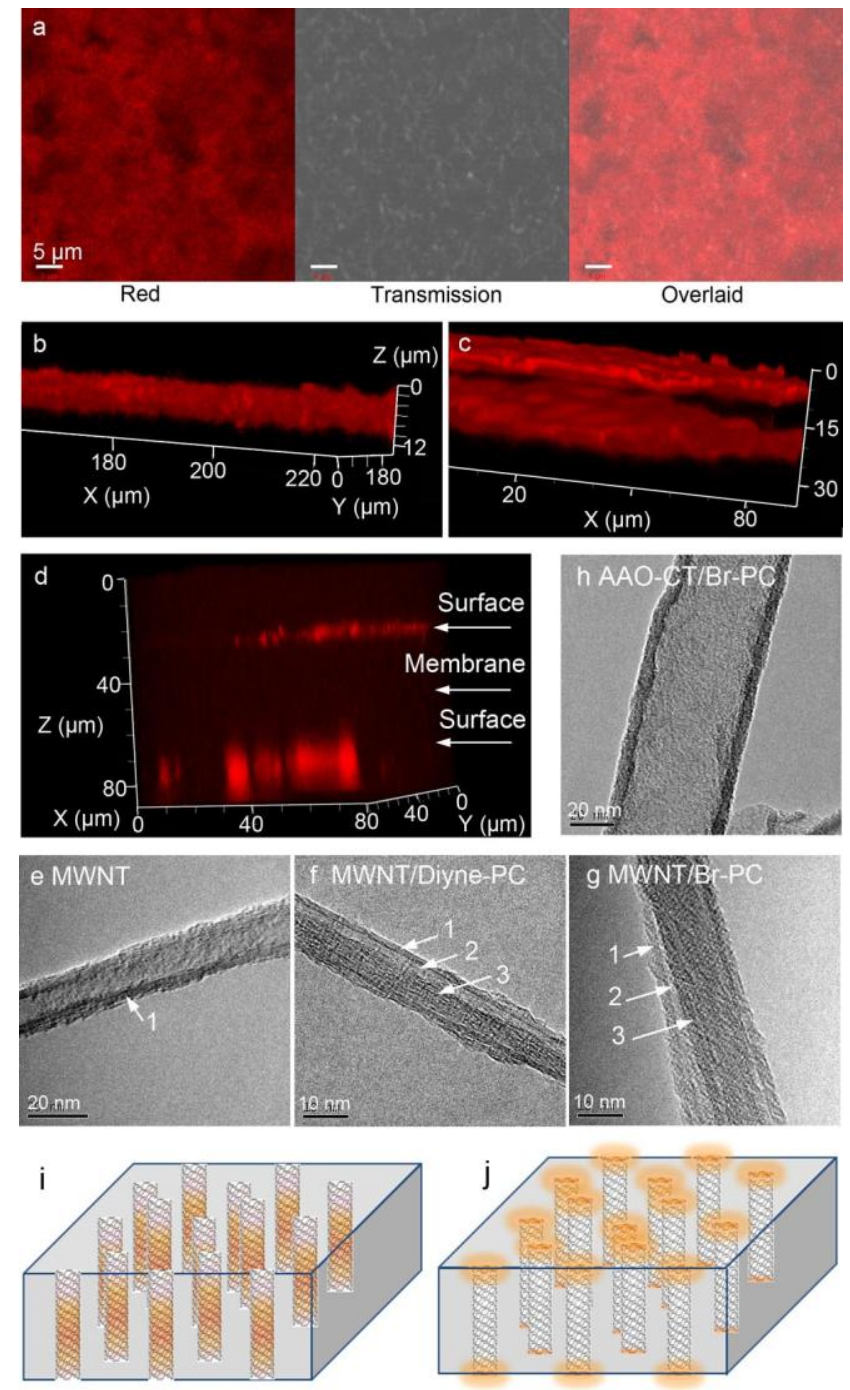

204 Fig. 3 Confocal fluorescence microscopy, TEM and schematic images of the MWNT/lipid and 205 AAO-CT/lipid membranes. (a) Confocal 2D (in red fluorescence, transmission and overlaid 206 channels) and (b-d) 3D images of the MWNT/DOPC (a, b) or AAO-CT/DOPC (d) composite 207 membrane. (c) Redistribution of lipids within the MWNT/DOPC composite membrane after 208 approximately five cycles of the ionic diffusion test. Red fluorescence comes from the Rh209 labeled lipid. (e-h) TEM images of MWNT, MWNT/Diyne-PC, MWNT/Br-PC and AAO- 
$210 \mathrm{CT} / \mathrm{Br}-\mathrm{PC}$ samples. Arrows 1-3 refer to the graphite layer, the gap and the encapsulated lipid

211 molecules, respectively. (i, j) Schematics representing the relative locations of fluorescent lipids

212 and CNTs within a MWNT/DOPC membrane, corresponding to (b) and (c), respectively.

\section{$213 \quad 3.2$ Influence from lipid decoration on ion transport rate through tubes}

214 Ion transport rates through tubes both with and without lipid decoration were measured based on

215 the concentration-driven diffusion of $\mathrm{KCl}$ across the membranes. The membranes were fixed

216 between a feed cell and a permeate cell within the diffusion unit, and the concentration gradient

217 between the two cells led to ion diffusion through tubes (Fig. 1b, c). Based on the time-

218 dependent increase in conductivity of the solution in the permeate cell, the ion transport rate

219 across the tubes was obtained. Furthermore, for the native MWNT-membrane, the conductivity

220 values were used to calculate the permeable pore area $\left(\mathrm{cm}^{2}\right)$ and density $\left(\# / \mathrm{cm}^{2}\right)$ which can

221 satisfactorily characterize the permeability of the membrane (Supporting Information, Section

222 S2). On the other hand, for the tubes with lipid decoration, the influence from lipid

223 functionalization was described by comparing the ion transport rate of each membrane before

224 and after lipid decoration, and consequently an average from more than three independent

225 samples was calculated. This is suspected to be more direct and accurate concerning the

226 deviation among various membrane samples.

227 Fig. 4 shows the time-lapse distribution of conductivity of the solution in the permeate cell,

228 corresponding to the ion transport rate through a membrane. For the native MWNT-membrane

229 (Fig. 4a, in black), the changes in conductivity are characterized with two typical stages: in Stage

230 I, the value increases quickly, referring to a fast transport of ions through tubes; then, in Stage II,

231 the value increases gradually with a linear dependence on time, indicating a dynamically- 
232 balanced transfer of ions in the tubes. Moreover, based on the steady state flux in Stage II, the

233 permeable pore density of our MWNT-membranes was calculated to be $2.2 \pm 0.2 \times 10^{8} \mathrm{~cm}^{-2}$,

234 which indicates a good permeability of the membrane (i.e., tubes in the membrane).
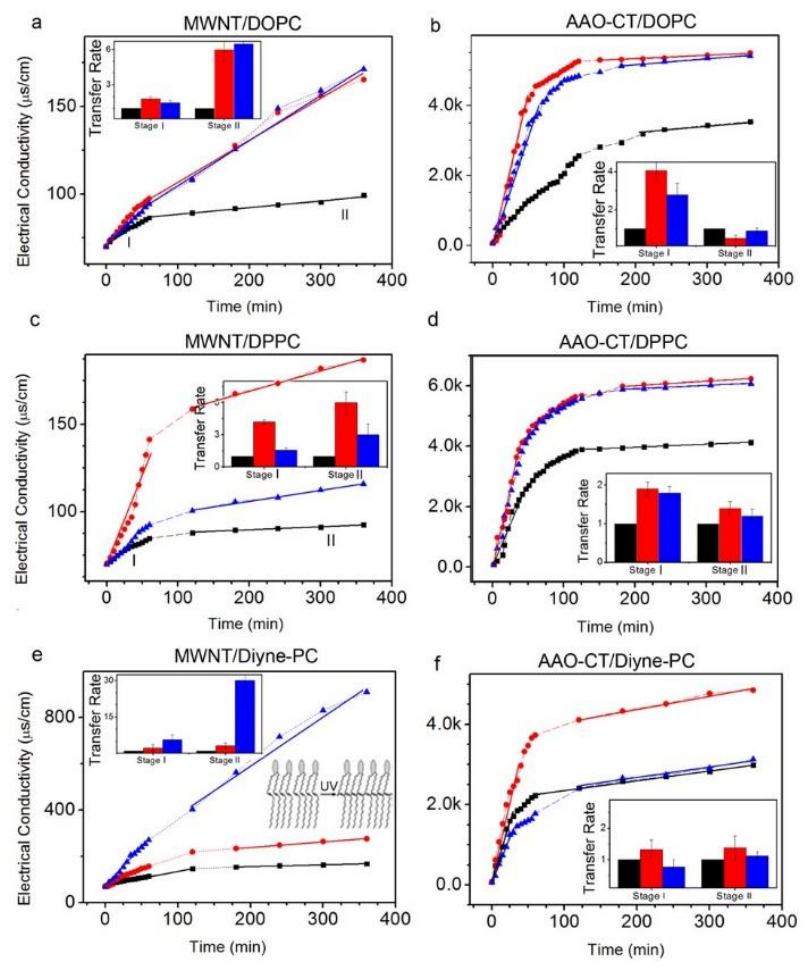

236 Fig. 4 Electrical conductivity changes with time for the diffusion solution during $\mathrm{KCl}$ transfer

237 process through typical MWNT/lipid (a, c, e) or AAO-CT/lipid (b, d, f) membranes. Insets, 238 corresponding ion transport rate calculated from the electrical conductivity distribution 239 (normalized by the value of the naked carbon membrane and averaged from 3 independent 240 samples). The black curve/histogram refers to the naked carbon membrane while the red (or 241 blue) one refers to that in the first (or repeated) ionic diffusion test; in (e, f), the red and blue 242 curves refer to the membrane before and after UV polymerization of Diyne-PC, respectively. 243 Right-inset in (e), schematic diagram demonstrating the polymerization of Diyne-PC lipids under 244 UV exposure. 
246 After DOPC decoration, obvious changes appear (Fig. 4a, in red). The evolution of

247 conductivity still shows a typical two-stage process, but the corresponding values experience a

248 significant increase. By fitting the conductivity profile, ion transport rates through tubes were

249 obtained. Before lipid decoration, the ion transport rates in stages I (concerning the almost linear

250 period before $60 \mathrm{~min}$ ) and II are $3.13 \times 10^{-10}$ and $4.68 \times 10^{-11}$ moles s $^{-1}$, respectively. After DOPC

251 decoration (in red), factors of $\sim 2$ (for Stage I, analyzed from more than three independent

252 samples) and $\sim 5$ (for Stage II) times increases occur (Fig. 4a, inset). Such improvements clearly

253 suggest that the assembly of lipids in the tube's interior is able to enhance ion transport through

254 CNTs.

255 Moreover, ion transport through tubes is significantly affected by the location and/or assembly

256 of lipids within tubes. Compared with the MWNT-membrane, the ion transport rate in the AAO-

257 CT-membrane only increases slightly during Stage I (from $\sim 2.75 \times 10^{-8}$ to $\sim 9.78 \times 10^{-8}$ moles s $^{-1}$,

258 referring to the typical membrane shown in Fig. 4b), while in Stage II, the value recovers to its

259 pre-lipid modification state (of around $2.2 \times 10^{-9}$ moles s ${ }^{-1}$ ). Note that in this case lipids mainly

260 accumulate at the tips of the tubes while small molecules remain inside of the tubes.

261 The type of lipid used in lipid decoration also has an impact on the enhancement effect on ion

262 transport through tubes. For the MWNT/DOPC composite membrane, it was found that $\underline{\text { in }}$

263 repeated ion-transport tests (after drying and rehydration of the membrane as stated in the

264 experimental section), the conductivity profile (Fig. 4a, in blue) almost overlaid with the initial

265 one (in red). This indicates that the enhancement effect on ion transport (i.e. the stability of lipid

266 decoration within tubes) is relatively stable. However, when we replaced DOPC with DPPC

267 which exists in gel phase at room temperature (Fig. 4c, black and red), it was found that the 
268 lipid-induced enhancement on ion transport occurred only in the first measurement (with an 269 enhancement rate being similar to that of DOPC); whereas in the following repeated cycles, the 270 ion transport rate recovers to the level of a case without lipid decoration (except for an 271 enhancement in Stage I ). In fact, even for DOPC decoration, after a repetition of 3-5 cycles, the 272 escape of DOPC molecules from the interior to the tips of the tubes (Fig. 3c), and the 273 corresponding recovery in ion transport rate, were observed. On the other hand, for the AAO274 CT-membrane, no obvious difference was observed when DOPC was replaced with DPPC, 275 indicating the stable accumulation of lipids at the tips of this type of tubes (Fig. 4d).

276 The stability of lipid decoration, especially inside the tubes, is crucial for practical 277 applications. To conquer this, another type of diacetylene lipid, Diyne-PC, was employed. It is 278 known that under UV radiation, the adjacent tails of Diyne-PC lipids tend to polymerize with 279 covalent bonds, leading to the formation of a continuous $\pi$ bond within the layer [38]. This 280 significantly improves the stability of the assembled lipid structure (Fig. 4e right-inset). By the 281 same concentration-driven method, Diyne-PC was incorporated into the MWNT-membrane, 282 which showed a similar distribution as that of DOPC inside the CNTs (Fig. S3a,b). UV 283 irradiation was then performed to polymerize the lipids. Fluorescence spectra of model 284 membranes before and after lipid polymerization are shown in Fig. S3c-e to confirm the 285 successful polymerization of lipids within membrane.

286 Fig. 4e shows the conductivity profiles for the condition of model MWNT-membrane, with 287 and without Diyne-PC incorporation, both before and after UV-polymerization. Before UV 288 irradiation, the influence of Diyne-PC decoration on steady ion transport rate was similar to 289 (although lower than) that of DOPC, with a 3-4 times enhancement (Fig. 4e in red); however, in 290 a sharp striking contrast, after polymerization, the steady ion transport rate was increased up to 
29120 times larger than that of the native membrane (Fig. 4e in blue). Furthermore, repeated tests

292 showed a good stability of such a promoted transport rate even after more than five cycles' test.

293 However, for the AAO-CT/Diyne-PC composite membrane (Fig. S3b), UV-polymerization had

294 little effect on the steady ionic transport (Fig. 4f).

$295 \quad 3.3$ Discussion

296 By an inter-diffusion method, lipids were successfully incorporated into CNTs. According to our 297 results, lipids can self-assemble inside or outside the tubes, probably depending on the surface 298 properties of the inner-walls of the tubes and the phase state of lipids. For MWNTs, the strong 299 affinity between the alkyl tails of lipid molecules and the hydrophobic aromatic plane of tubes 300 facilitates the localization of lipids within the interior of the tubes (Supporting Information. 301 Section S3). However, for AAO-CTs, the hydrophilic surface of the amorphous carbon layer 302 makes it difficult for lipids to stay inside the tubes, although it is much easier for an assembled 303 lipid layer to attach to a less curved surface (i.e. AAO-CTs with a larger size) in comparison with

304 a highly curved one (i.e. MWNT with a smaller size; Supporting Information, Section S4).

305 Furthermore, in comparison with gel-phase DPPC, the flexible tails of liquid-phase DOPC 306 promotes the assembly and localization of lipids on the highly-curved inner surface of CNTs as a 307 result of a much lowered energy cost for layer bending (Supporting Information, Section S4).

308 It is clearly demonstrated that the assembly of lipids inside the MWNTs significantly 309 facilitates the ion transport through the tubes, on the basis of confocal, TEM and ion transport 310 tests. Moreover, polymerization of Diyne-PC inside the tubes further enhances the steady ion 311 transport compared with that of DOPC/DPPC; this is probably due to the more uniform and 312 stable distribution of lipid molecules caused by inter-molecule binding between adjacent Diyne-

313 PCs. In addition, accumulation of lipids at the tips of the CNTs could also boost the ion transport 
314 rate at the initial stage, although without much influence on the steady ion transport process.

315 These results all promise the regulation of ion transportation behaviors through CNTs for 316 practical applications.

317 The influence of lipid decoration on ion transport is suspected to be associated with the 318 complicate ion-lipid interactions, which could create a preferred distribution of ions near the 319 zwitterionic lipid headgroups [39-41]. Electroneutral or negatively charged molecules were also 320 used instead of the zwitterionic lipids for MWNT decoration, which, however, reduced the ion

321 transport rate (Fig. S4). In this regard, the accumulated lipids at tube-tips would increase the 322 local ionic concentration at the tube entrance, and consequently enhance the ion transport under 323 the effect of flow during the initial period (i.e. Stage I). In contrast, the incorporation and 324 continuous localization of lipids along the tubes might provide successional binding sites for ions 325 with lipid headgroups, which could work as a highway for the ion transport throughout the whole 326 tube, and thus significantly increase the steady ion transport rate (although the pore size might 327 decrease due to lipid incorporation). The remarkable increase in ion transport due to the 328 polymerized Diyne-PC decoration further confirms this speculation.

\section{4. Conclusion}

330 In this study, we prepared two types of vertically-aligned CNT membranes composed of 331 MWNTs or AAO-based amorphous CNTs, and investigated the influence of lipid decoration on 332 the ion transport properties through the inner core of the nanotubes via macroscopic transport 333 measurements. Concentration-driven diffusion was employed for the incorporation of lipids 334 within the tubes. Confocal imaging and TEM observation indicated the continuous distribution 335 of lipids inside the MWNTs, probably due to the hydrophobic interaction between the alkyl tails 
336 of lipids and the aromatic wall plane of MWNT. In contrast, for the AAO-CT-membrane, lipids

337 tended to accumulate at the two sides of the membrane, likely at the tips of the carbon tubes. Ion

338 transport tests demonstrated an enhancement in the steady ion transport rate through MWNTs

339 due to lipid incorporation, by approximately 5 times for DOPC or DPPC, 3-4 times for Diyne-

340 PC, and up to 20 times for Diyne-PC after polymerization. Furthermore, the accumulation of

341 lipids at the tips of the carbon tubes (both MWNTs and AAO-CTs) accelerated the ion transport

342 during the initial stage, but hardly influenced the steady transport rate of ions. The increase in

343 local ionic concentration due to the binding of ions to zwitterionic headgroups of the decorated

344 lipids is supposed to be one of the key factors for the enhanced ion transport rate. Our results

345 provide promising possibilities for selective and high-efficiency transport of CNTs for separation

346 and sensing applications [16], after further functionalization of lipids.

\section{Acknowledgments}

349 This work was financially supported by the National Science Foundation of China (Nos. $35091427302,21422404,21374074$, U1532108 and 11474155). The authors sincerely thank Prof.

351 Guowen Meng (Institute of Solid State Physics, Chinese Academy of Sciences) for providing the

352 AAO-based CT-membrane. The authors also thank the Small Angle X-ray Scattering Station

353 (BL16B) and X-ray Diffraction Station (BL14B) at Shanghai Synchrotron Radiation Facility

354 (SSRF) for sample characterizations.

355 Appendix A. Supplementary data

356 Confocal z-stack images of the MWNT/DOPC and AAO-CT/DOPC composite membranes;

357 synchrotron SAXS pattern of the AAO-CT/DOPC membrane; confocal 3D images and PL 358 profiles of the MWNT/Diyne-PC and AAO-CT/Diyne-PC membranes; ion transport through 
MWNT membranes after PS-PAA or calcein decoration; estimation of the permeable pore area

360 and density from $\mathrm{KCl}$ diffusion measurements; interaction energy analysis between components

361 of a lipid-decorated MWNT system; energetic analysis of DOPC and DPPC assemblies in

362 MWNTs. These materials can be found, in the online version, at ...

\section{REFERENCES}

364 [1] Hong GS, Diao S, Antaris AL, Dai HJ. Carbon nanomaterials for biological imaging and 365 nanomedicinal therapy. Chem Rev 2015; 115(19): 10816-10906.

366 [2] Mehra NK, Jain NK. Multifunctional hybrid-carbon nanotubes: new horizon in drug 367 delivery and targeting. Journal of Drug Targeting. J Drug Target 2016; 24(4): 294-308.

368 [3] Sajid MI, Jamshaid U, Jamshaid T, Zafar N, Fessi H, Elaissari A. Carbon nanotubes from 369 synthesis to in vivo biomedical applications. Int J Pharm 2016; 501(1-2): 278-299.

370 [4] Mehra NK, Jain AK, Lodhi N, Raj R, Dubey V, Mishra D, et al. Challenges in the use of 371 carbon nanotubes for biomedical applications. Crit Rev Ther Drug Carrier Syst 2008; 25(2): $372 \quad 169-206$.

373 [5] Majumder M, Stinchcomb A, Hinds BJ. Towards mimicking natural protein channels with 374 aligned carbon nanotube membranes for active drug delivery. Life Sci 2010; 86(15-16): 563375568.

376 [6] Hinds B. Dramatic transport properties of carbon nanotube membranes for a robust protein 377 channel mimetic platform. Curr Opin Solid St M 2012; 16(1): 1-9.

378 [7] Majumder M, Chopra N, Hinds BJ. Mass transport through carbon nanotube membranes in 379 three different regimes: ionic diffusion and gas and liquid flow. ACS Nano 2011; 5: 3867-3877. 
[8] He Z, Zhou J, Lu X, Corry B. Bioinspired graphene nanopores with voltage-tunable ion 381 selectivity for $\mathrm{Na}^{+}$and $\mathrm{K}^{+}$. ACS Nano 2013; 7(11): 10148-10157.

382 [9] He Z, Corry B, Lu X, Zhou J. A mechanical nanogate based on a carbon nanotube for 383 reversible control of ion conduction. Nanoscale 2014; 6: 3686-3694.

384 [10] Whitby M, Quirke N. Fluid flow in carbon nanotubes and nanopipes. Nat Nanotechnol $385 \quad 2007 ; 2: 87-94$.

386 [11] Majumder M, Chopra N, Andrews R, Hinds BJ. Enhanced flow in carbon nanotubes. 387 Nature 2005; 438: 44.

388 [12] Hummer G, Rasaiah JC, Noworyta JP. Water conduction through the hydrophobic 389 channel of a carbon nanotube. Nature 2001; 414: 188-190.

390 [13] Rivera JL, Starr FW. Rapid transport of water via a carbon nanotube syringe. J Phys 391 Chem C 2010; 114: 3737-3742.

392 [14] Cohen-Tanugi D, Grossman JC. Water Desalination across Nanoporous Graphene. Nano 393 Lett 2012; 12: 3602-3608.

394 [15] Pham TA, Golam Mortuza SM, Wood BC, Lau EY, Ogitsu T, Buchsbaum SF, et al. Salt 395 solutions in carbon nanotubes: the role of cation- $\pi$ interactions. J Phys Chem C 2016; 120(13): $396 \quad 7332-7338$.

397 [16] Hinds BJ, Chopra N, Rantell T, Andrews R, Gavalas V, Bachas LG. Aligned multiwalled 398 carbon nanotube membranes. Science 2004; 303(5654): 62-65. 
[17] Moskowitz I, Snyder MA, Mittal J. Water transport through functionalized nanotubes with

400 tunable hydrophobicity. J Chem Phys 2014; 141(18): 18C532.

401 [18] Yu M, Funke HH, Falconer JL, Noble RD. Gated ion transport through dense carbon 402 nanotube membranes. J Am Chem Soc 2010; 132: 8285-8290.

403 [19] Pan Y, Wu Q, Weng Y, Zhang X, Yang Z, Meng J, et al. Declined ionic flux through the 404 nano-pores of vertically aligned carbon nanotubes filled with PNIPAm hydrogel. J Mater Chem 405 A 2015; 3: 11111-11116.

406 [20] Zhu Y, Guo X. Shao Q, Wei M, Wu X, Lu L, et al. Molecular simulation study of the 407 effect of inner wall modified groups on ionic hydration confined in carbon nanotube. Fluid Phase 408 Equilibria 2010; 297: 215-220.

$409 \square 21]$ He Z, Zhou J, Lu X, Corry B. Ice-like water structure in carbon nanotube $(8,8)$ induces 410 cationic hydration enhancement. J Phys Chem C 2013; 117: 11412-11420.

411 [22] Kim SW, Kim T, Kim YS, Choi HS, Lim HJ, Yang SJ, et al. Surface modifications for the 412 effective dispersion of carbon nanotubes in solvents and polymers. Carbon 2012; 50(1): 3-33.

413 [23] Wu Y, Hudson JS, Lu Q, Moore JM, Mount AS, Rao AM, et al. Coating single-walled 414 carbon nanotubes with phospholipids. J Phys Chem B 2006; 110: 2475-2478.

415 [24] Diao S, Blackburn JL, Hong GS, Antaris AL, Chang JL, Wu JZ, et al. Fluorescence 416 imaging in vivo at wavelengths beyond $1500 \mathrm{~nm}$. Angew Chem Int Edit 2015; 54(49): 1475841714762. 
418 [25] Liang C, Diao S, Wang C, Gong H, Liu T, Hong G, et al. Tumor metastasis inhibition by 419 imaging-guided photothermal therapy with single-walled carbon nanotubes. Adv Mater 2014; $420 \quad 26(32): 5646-5652$.

421 [26] Hong G, Diao S, Chang J, Antaris AL, Chen C, Zhang B, et al. Through-skull 422 fluorescence imaging of the brain in a new near-infrared window. Nat Photonics 2014; 8(9): $423 \quad 723-730$.

424 [27] Islam MF, Rojas E, Bergey DM, Johnson AT, Yodh AG. High weight fraction surfactant 425 solubilization of single-wall carbon nanotubes in water. Nano Lett 2003; 3(2): 269-273.

426 [28] Giulianini M, Waclawik ER, Bell JM, De Crescenzi M, Castrucci P, Scarselli M, et al. 427 Regioregular poly(3-hexyl-thiophene) helical self-organization on carbon nanotubes. Appl Phys $428 \quad$ Lett 2009; 95(1): 013304.

429 [29] Maatta J, Vierros S, Sammalkorpi M. Controlling carbon-nanotube-phospholipid 430 solubility by curvature-dependent self-assembly. J Phys Chem B 2015; 119(10): 4020-4032.

431 [30] Arai N, Yasuoka K, Zeng XC. Phase diagrams of confined solutions of 432 dimyristoylphosphatidylcholine (DMPC) lipid and cholesterol in nanotubes. Microfluid 433 Nanofluid 2012; 14(6): 995-1010.

434 [31] Arai N, Yasuoka K, Zeng XC. Self-assembly of surfactants and polymorphic transition in 435 nanotubes. J Am Chem Soc 2008; 130: 7916-7920.

436 [32] Khlobystov AN, Britz DA, Briggs GAD. Molecules in carbon nanotubes. Accounts Chem 437 Res 2005; 38(12): 901-909. 
439 in inorganically filled carbon nanotubes: successes and challenges. Sc Technol Adv Mater 2010;

440 11(5): 054501.

441 [34] Muter D, Shin T, Deme B, Fratzl P, Paris O, Findenegg GH. Surfactant self-assembly in 442 cylindrical silica nanopores. J Phys Chem Lett 2010; 1(9): 1442-1446.

443 [35] Liao G, Pan Y, Wu Q, Li S, Weng Y, Zhang X, et al. A novel method to encapsulate a Au 444 nanorod array in $15 \mathrm{~nm}$ radius multiwalled carbon nanotubes. Nanoscale 2014; 6: 14872-14876.

445 [36] Liu Z, Liao G, Li S, Pan Y, Wang X, Weng Y, et al. Efficient encapsulation of conducting 446 polyaniline chains inside carbon nanotubes: a new strategy to prepare endohedral CNT materials. 447 J Mater Chem A 2013; 1(42): 13321-13327.

448 [37] Jani AMM, Losic D, Voelcker NH. Nanoporous anodic aluminium oxide: advances in 449 surface engineering and emerging applications. Prog Mater Sci 2013; 58(5): 636-704.

450 [38] Yuan B, Hu S, Lu N, Xu F, Zhou K, Ma Y, et al. Electrical bistability in self-assembled 451 hybrid multilayers of phospholipid and nanoparticles. Nanotechnology 2011; 22(31): 315303.

452 [39] Vacha R, Berkowitz ML, Jungwirth P. Molecular model of a cell plasma membrane with 453 an asymmetric multicomponent composition: water permeation and ion effects. Biophys J 2009; 454 96(11): 4493-4501.

455 [40] Klasczyk B, Knecht V. Validating affinities for ion-lipid association from simulation 456 against experiment. J Phys Chem A 2011; 115(38): 10587-10595.

457 [41] Seelig J, MacDonald PM, Scherer PG. Phospholipid head groups as sensors of electric 458 charge in membranes. Biochemistry 1987; 26(24): 7535-7541. 
a<smiles>CCCCCCCCC=CCCCCCCCCCCCCCC(=O)OCC(COP(=O)([O-])OCC[N+](C)(C)C)OC(=O)CCCCCCCCCC</smiles><smiles>[2H]P(C)(=O)OC[C@H](COP(=O)([O-])OCC[N+](C)(C)C)OC(=O)CCCCCCCCCCCCCCCCCCCCCCCCCCC</smiles><smiles>CCCCCCCCCCCCCCCCCCCCC(=O)OCC(COP(=O)([O-])OCC[N+](C)(C)C)OC(=O)CCCCCCCC(Br)Br</smiles>

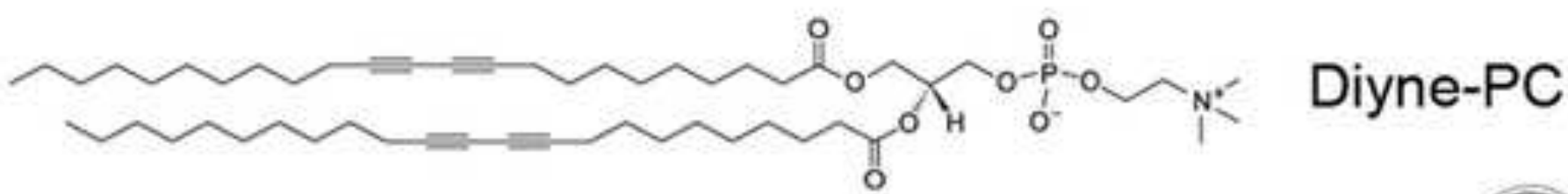

b

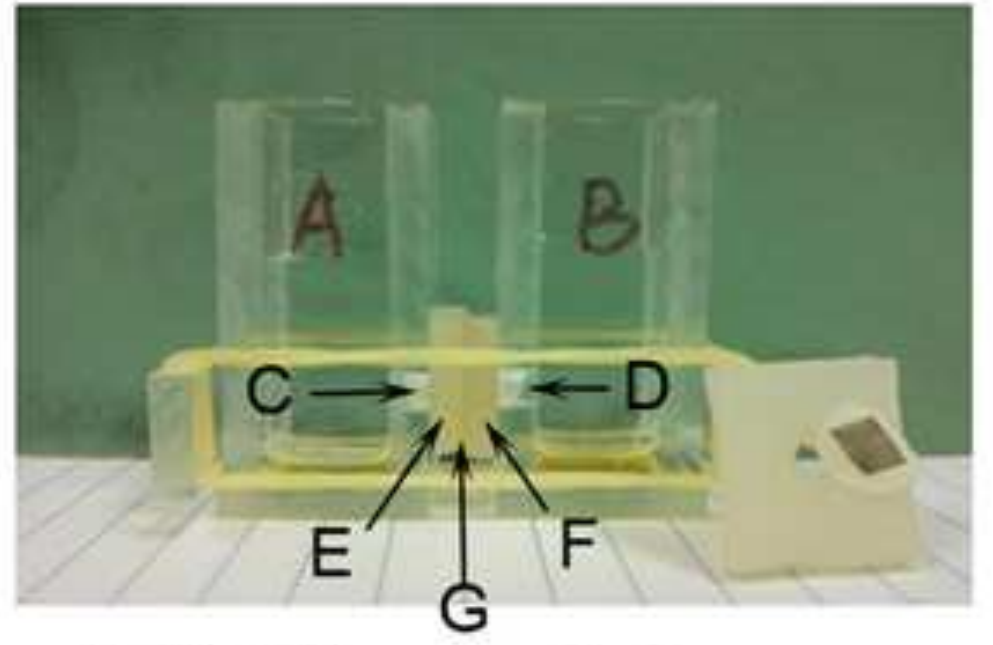

A, B: cell
C, D: pore
E, F: spacer G: membrane

\section{Membrane}

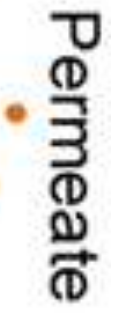

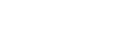




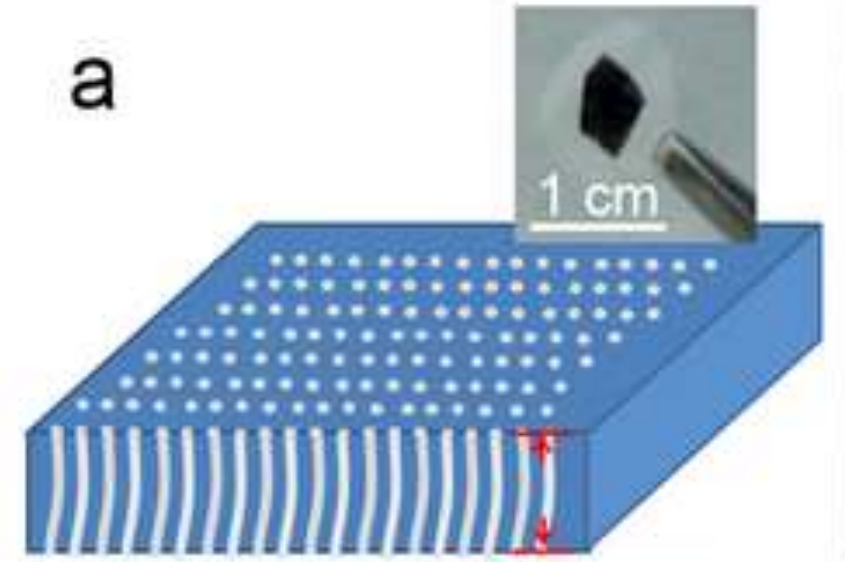

Thickness

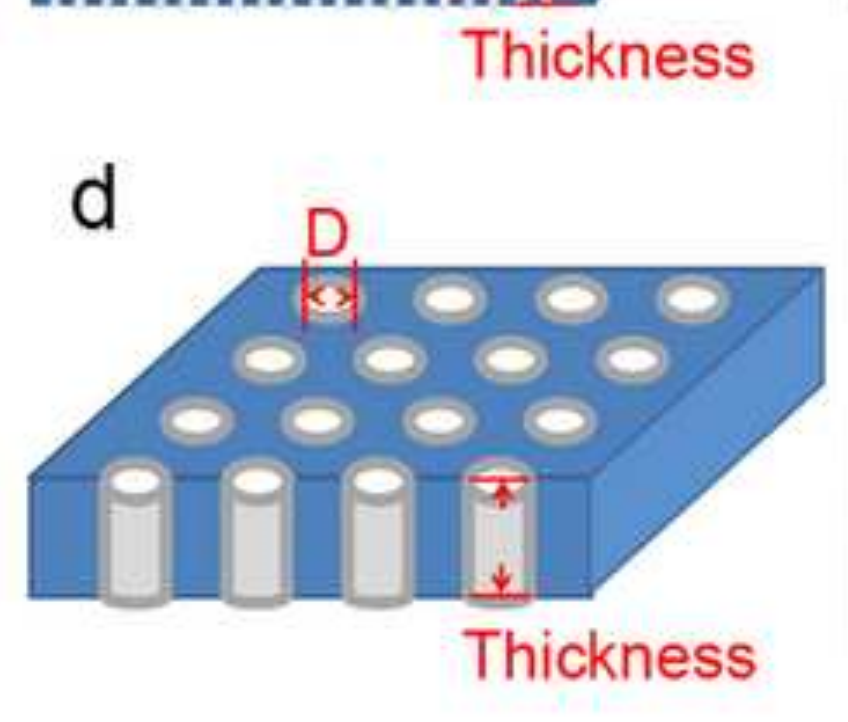

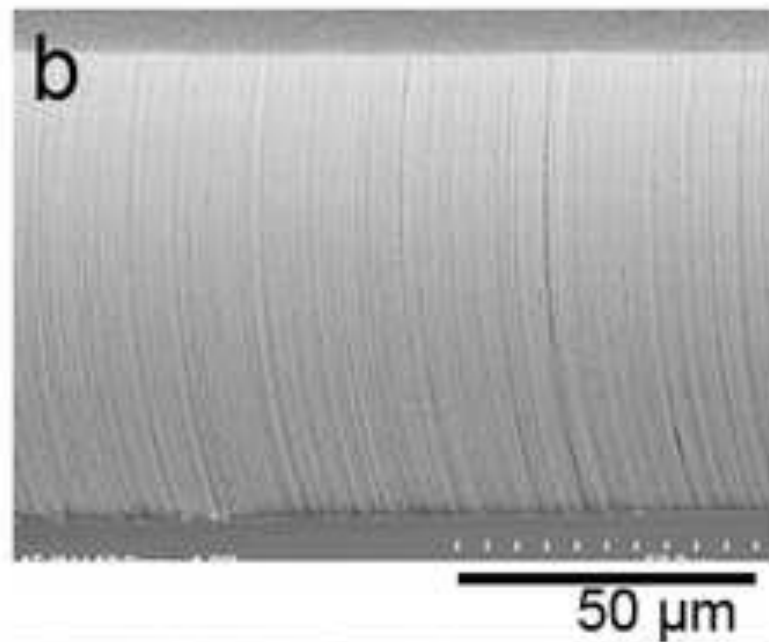
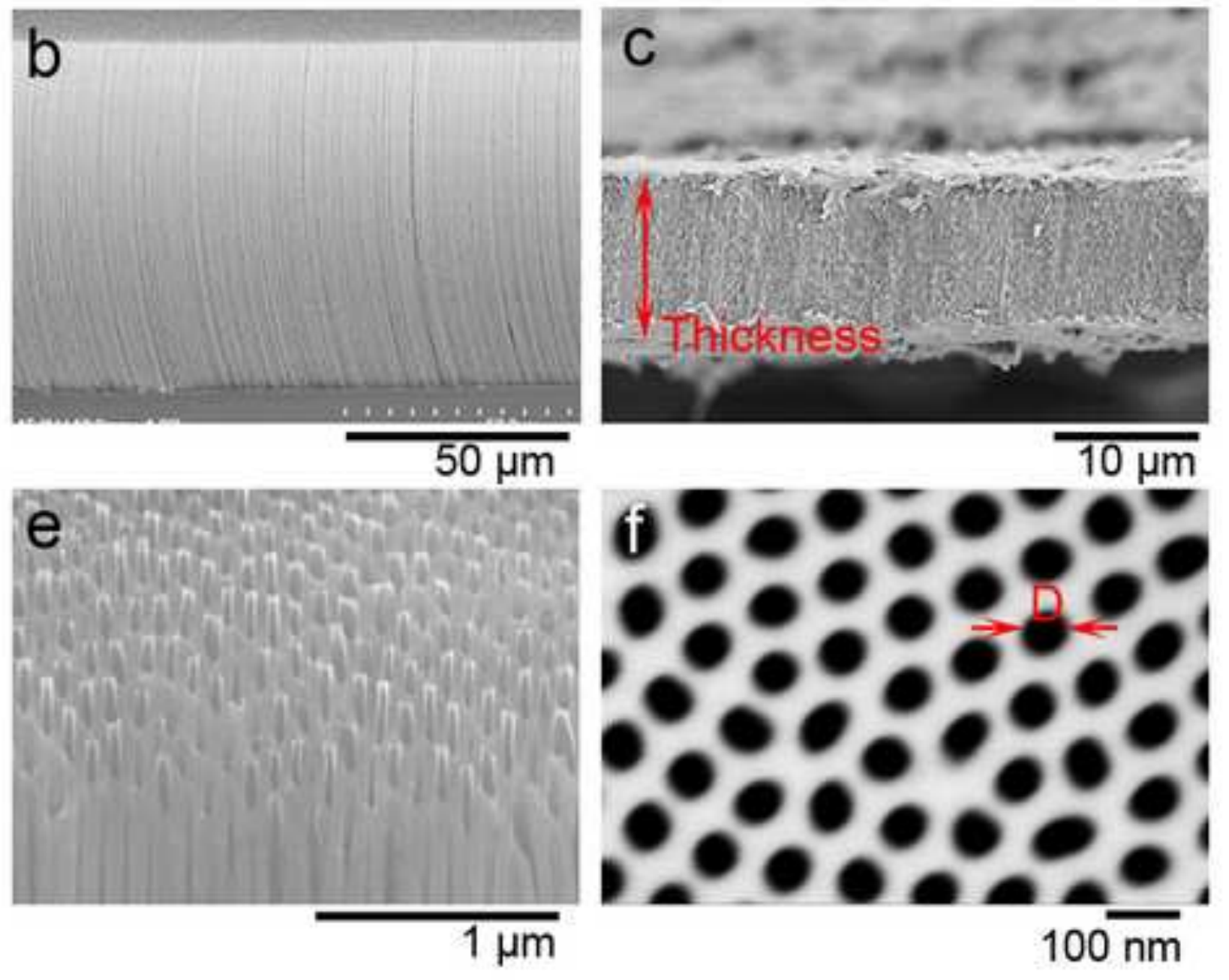


\section{Click here to download high resolution image}
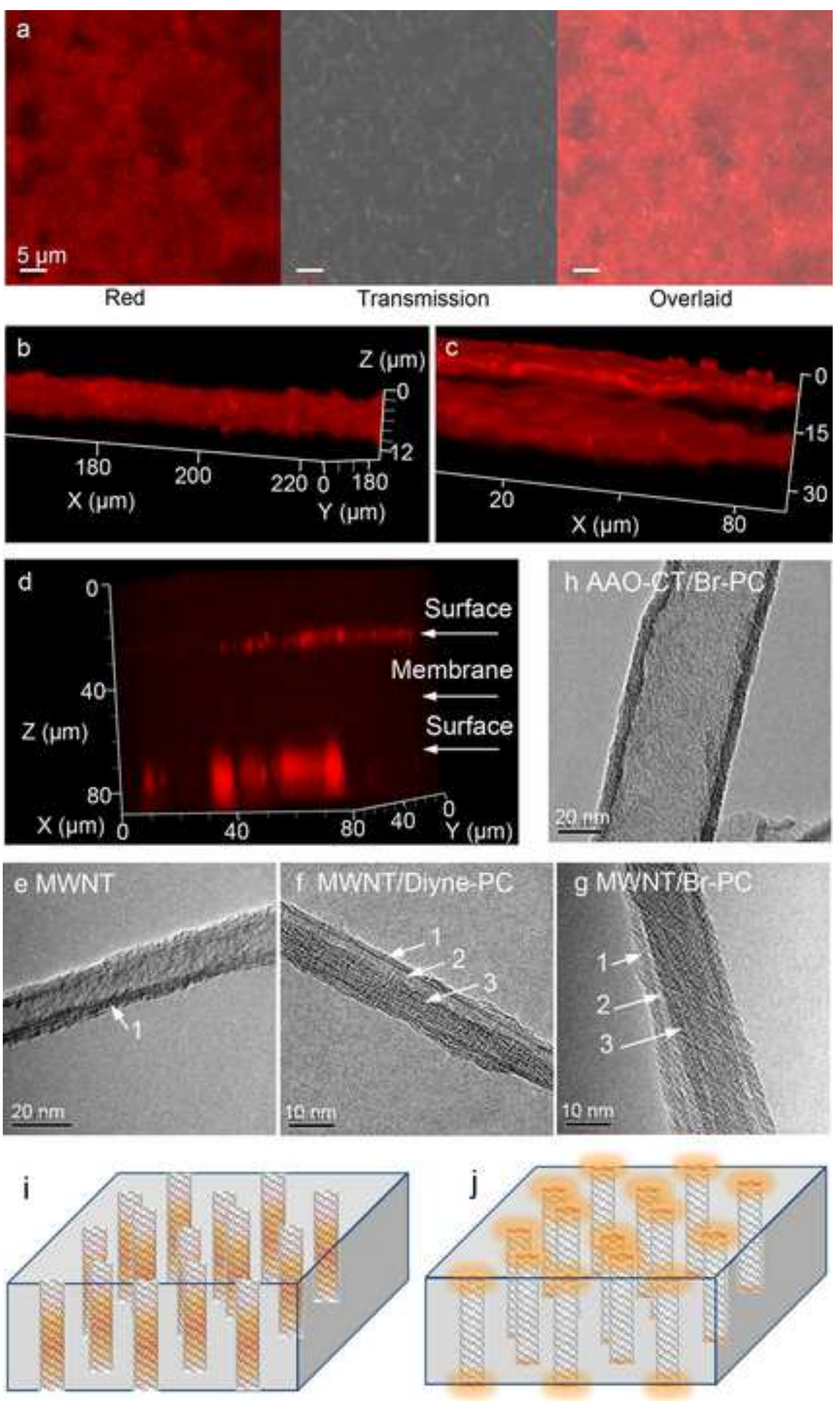

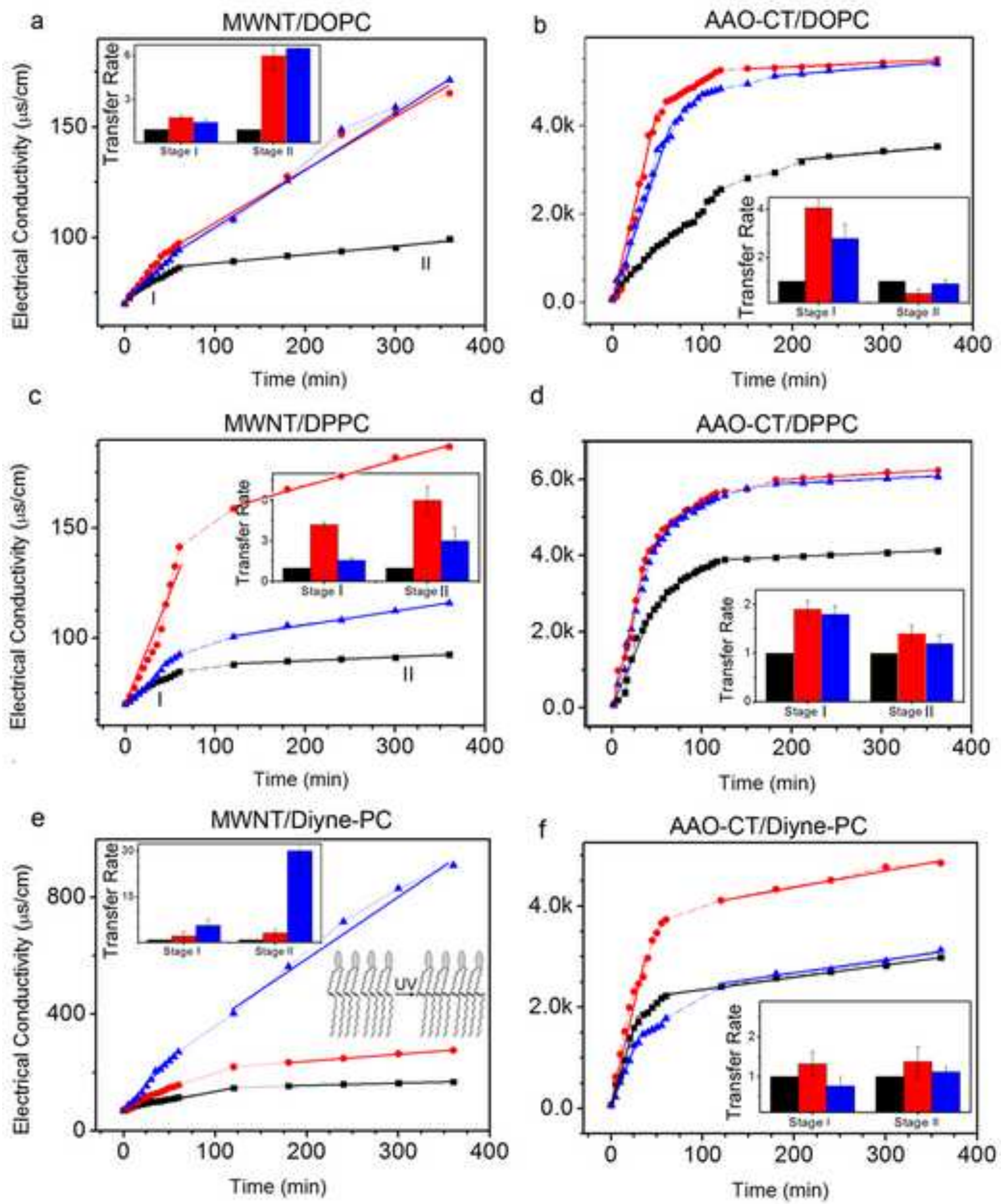
Supplementary Material
Click here to download Supplementary Material: ESI_marked-1011.docx

Click here to download Supplementary Material: Es_marked-1011.docx

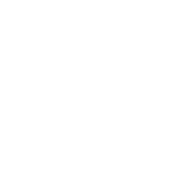

$\sqrt{2}$

(1)

(1)

(1)

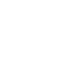
$\sqrt{10}$ (1) (1)

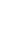
(1) . . . . . . . . . . . . . . . . . .

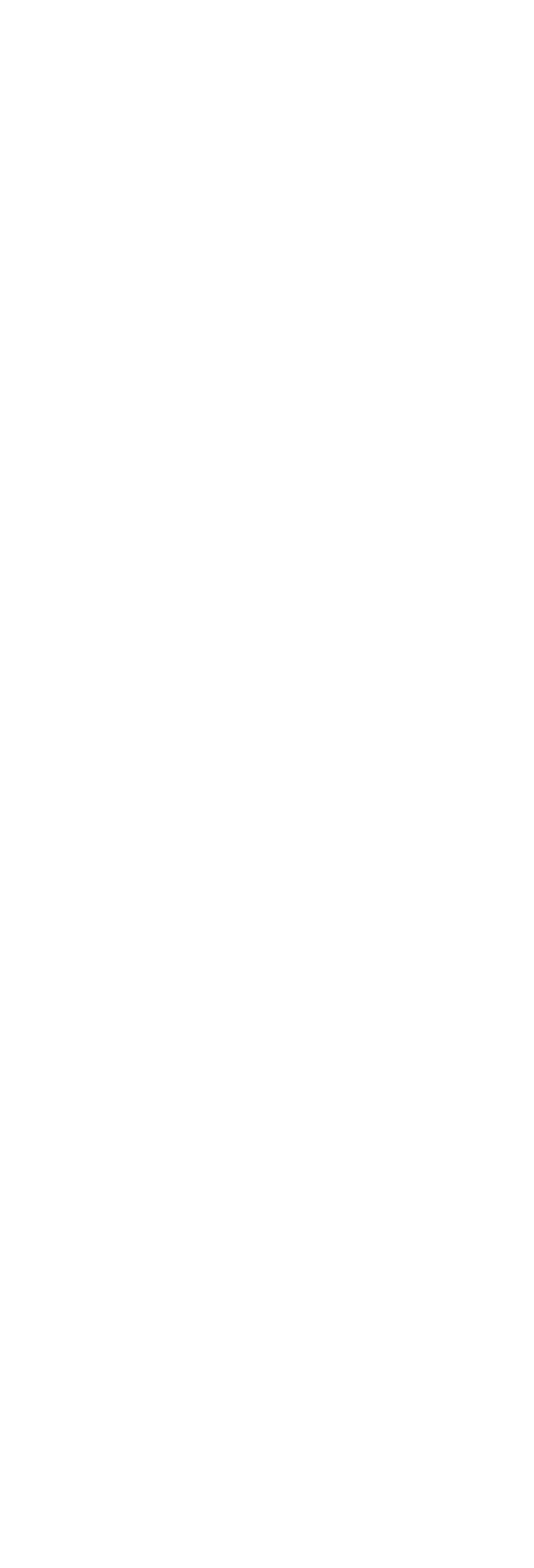

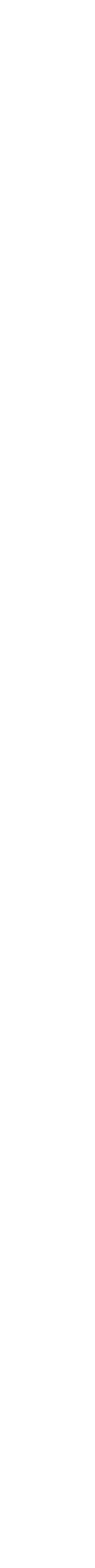
(1) 